
\title{
Besearch S Surare \\ Design and modeling of a planar graphene structure as a terahertz cyclotron radiation source
}

Jordan Planillo ( $\sim$ jordan.planillo@nps.edu )

Naval Postgraduate School

\section{Fabio Alves}

Naval Postgraduate School

\section{Research Article}

Keywords: planar graphene structure, Larmor formulation, terahertz cyclotron radiation

Posted Date: February 18th, 2021

DOl: https://doi.org/10.21203/rs.3.rs-181614/v1

License: (a) (i) This work is licensed under a Creative Commons Attribution 4.0 International License. Read Full License

Version of Record: A version of this preprint was published at Scientific Reports on August 5th, 2021. See the published version at https://doi.org/10.1038/s41598-021-95502-9. 


\title{
Design and modeling of a planar graphene structure as a terahertz cyclotron radiation source
}

\author{
Jordan Planillo ${ }^{1 *}$ \\ Fabio Alves ${ }^{1}$ \\ ${ }^{1}$ Naval Postgraduate School \\ 1 University Circle, Monterey, CA 93943 \\ jordan.planillo@nps.edu
}

\begin{abstract}
With incredibly high carrier mobility and saturation velocity, graphene would be an ideal candidate for a miniaturized solid-state cyclotron radiation source. A planar semicircular graphene arc geometry was investigated for emission in the $0.5 \mathrm{THz}$ to $1.5 \mathrm{THz}$ range. Analytical studies, confirmed by finite element simulations, show that the emitted $\mathrm{THz}$ frequencies are inversely proportional to the arc radius given a fixed charge-carrier velocity. The simulations show that the desired frequency spectrum can be obtained with design radii ranging from $50 \mathrm{~nm}$ to $150 \mathrm{~nm}$. Interestingly, the radiated spectrum is independent of the frequency of the stimulation of the graphene nano-arcs. The simulations also indicate that the total output power correlates well with the Larmor formulation. The device is expected to emit $1 \mathrm{nW} / \mathrm{cm}^{2}$, which confirms the findings of existing research in this field. Such a design could yield a scalable and cost-effective THz source.
\end{abstract}

\section{Introduction}

Sixteen years since its discovery [1] [2] [3], graphene has been touted as a super material known for its excellent mechanical, thermal, and electrical properties. Promising applications range from a new construction material [4] to a room temperature superconductor [5]. While most of the electronics applications for graphene involve its use as a field effect transistor to eventually replace silicon for computing applications, few have explored its use in radio frequency (RF) emissions [6]. Much of graphene's RF applications have involved design and construction of conventional RF components such as transmission lines, waveguides [7], and antennas [8] [9]. More recently, graphene's high carrier mobility [10] and saturation velocity [11] has been explored for its application as a solid state implementation of a free electron laser (FEL) and associated devices such as the wiggler [12] [13].

The use of graphene in RF emitter applications is primarily due to its high carrier mobility, high saturation velocity, and its tunable band gap. Highly conventional approaches and applications recreate existing RF structures in graphene - namely the patch antenna [8]. The monolayer thickness of graphene makes it highly desirable for compact form factor applications. In another such application, a graphene substrate is used to enhance the response and tunability of plasmonic antennas [9]. By applying a back-gate field to the graphene substrate and altering 
the graphene's bandgap the antenna can respond to a much wider span of incident wavelengths compared to the same photoconductive structure without graphene.

Less conventional RF emitter applications envision graphene as a substrate which can mimic the behavior of vacuum based particle accelerator devices such as the cyclotron, FEL, or wiggler. Models of corrugated graphene show that under a constant DC bias, electromagnetic radiation can be emitted with a frequency equal to the carrier drift velocity divided by the corrugation period [12]. With this approach, emissions at $\mathrm{THz}$ frequencies are possible that are not dependent on the existence of THz switching technologies. Experimental implementations of corrugated graphene have shown that $\mathrm{THz}$ emission is possible [13]. Such an approach involves etching the periodic trenches onto a Ge substrate and transferring the graphene onto the patterned substrate. The graphene contours the substrate resulting in the desired corrugation. Expected power output for this device ranges from $1 \mathrm{pW} / \mathrm{cm}^{2}$ to $10 \mathrm{nW} / \mathrm{cm}^{2}$.

Other cyclotron based radiation methods involve radiative transitions between Landau levels in graphene [14] [15] [16]. Such methods subject graphene to a magnetic field such that quantized cyclotron orbits via the quantum Hall effect occur. Optical stimulus, by means of a laser, pumps electrons from the valence band to the conduction band of the graphene at energies set by graphene's dispersion relation and by the Landau levels. Photon emission via transition between Landau levels occurs at Far-Infrared (FIR) and terahertz frequencies.

In this paper, the application of graphene as a miniaturized solid-state cyclotron radiation source in the form of semicircular arcs is explored. A design geometry should satisfy the desired frequency output range from $0.5 \mathrm{THz}$ to $1.5 \mathrm{THz}$ while being scalable with existing semiconductor processing methods. Modeling begins with a simple rotating dipole model as an idealized scenario for the device to obtain estimates for the power spectrum and radiation pattern. A finite element model that better represents the actual structure and allows for finite width and electronic contacts is also developed. To facilitate future device scalability, design considerations for higher density device arrays are also considered.

We propose here a device that lies on a flat substrate plane in which graphene is patterned as a semicircular arc. Such a device will produce cyclotron radiation by charges traversing along the arc. Such a structure would be highly compatible with existing semiconductor processing techniques, especially in terms of scalability and high density layouts (Figure 1). Structures like this are now feasible since graphene manufacturing processes are mature enough to where whole wafers of single layer graphene (grain size $\sim 10 \mathrm{um}$ ) can be purchased [17]. To model such a device and obtain performance estimates, the first assumption is that all the available charge carriers in the semicircular arc are concentrated in a singular point charge at the arc's inner radius $\left(r_{\text {arc }}\right)$. Next, the singular point charge moves at graphene's saturation velocity $\left(v_{s a t}\right)$ of $4.25 \times 10^{7} \mathrm{~cm} / \mathrm{s}$ [11]. For a sufficiently large and spontaneously applied field, the charge carriers in the graphene will accumulate in a line spanning the width of the graphene sample [18], hence a sufficiently short width would give the appearance of a moving point charge. 


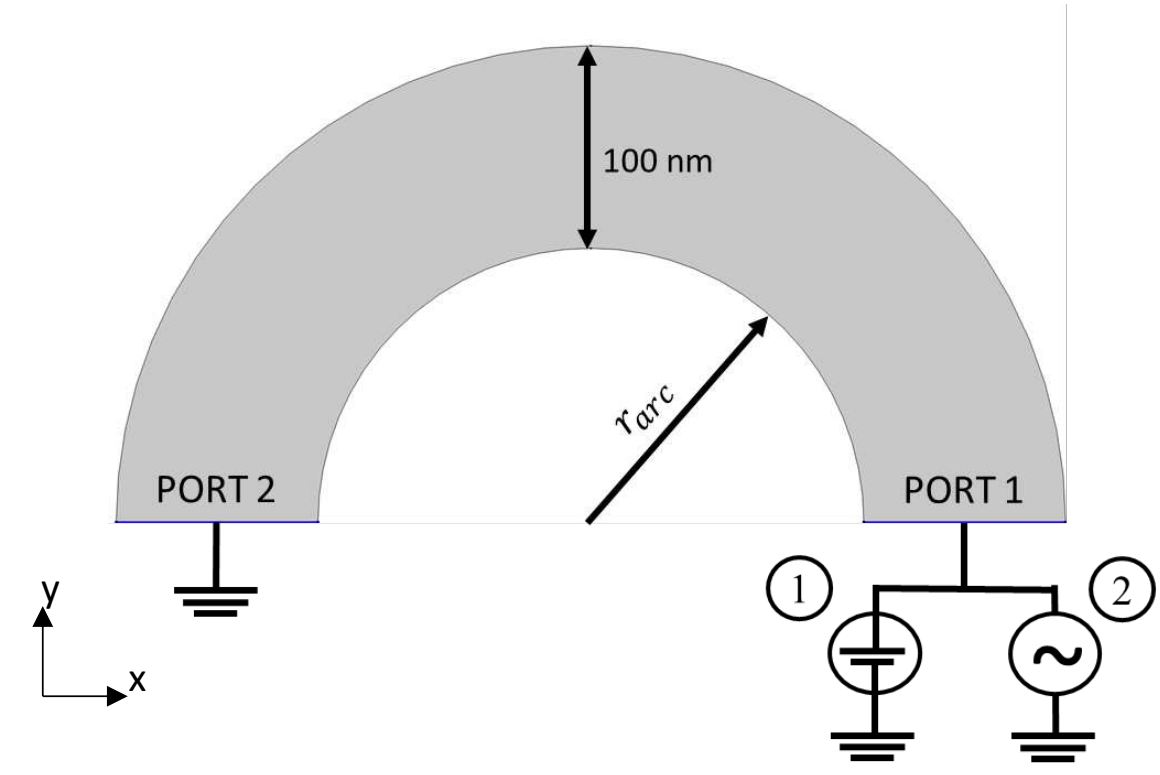

Figure 1 Schematic setup of the graphene arcs. In the dipole model, all of the arc's charges are concentrated at a single point and traverse a semicircular trajectory at a radius $r_{\text {arc. }}$ The motion of the charges is expected to produce cyclotron radiation at frequencies inversely proportional to rarc. For the finite element DC simulation (1), a constant $1 \mathrm{~V}$ was applied. For the finite element RF simulation (2), signals at $4 \mathrm{GHz}, 10 \mathrm{GHz}$, and $40 \mathrm{GHz}$ of $1 \mathrm{~V}$ amplitude were applied to port 1.

While graphene's saturation velocity offers superior speed compared to most semiconducting materials, this velocity is still nowhere close to the relativistic speeds in free electron laser, wigglers, or synchrotron light sources [19]. This implementation of a solid state cyclotron radiation source operates in the non-relativistic regime and thus can be initially modeled as a rotating dipole [20]. When reduced to this classic problem, this device is expected to emit radiation at a frequency equivalent to its angular velocity at a power given by the Larmor formula

$$
P=\frac{q^{2} a^{2}}{6 \pi \epsilon_{0} c^{3}}
$$

where $q$ is the effective single point charge of the graphene arc, $a$ is the charge's centripetal acceleration ( $\left.a=v_{\text {sat }}^{2} / r_{\text {arc }}\right), \epsilon_{0}$ is the vacuum permittivity, and $c$ is the speed of light in vacuum.

For fixed particle velocity $\mathrm{v}_{\text {sat }}$ and frequency $f$, the required arc radius is $r_{\text {arc }}=v_{\text {sat }} / 2 \pi f$. The relation of $r_{a r c}$ to frequency is displayed in Figure 1. For design frequencies $0.5 \mathrm{THz}$ and 1.0 $\mathrm{THz}$ they are $135 \mathrm{~nm}$ and $67 \mathrm{~nm}$ respectively. An arc width of $100 \mathrm{~nm}$ is chosen as it is sufficiently wide to avoid quantized current behaviors in the graphene nanoribbon regime [21] [22]. These parameters can be easily manufactured with existing semiconductor processing methods while also being within the constraints of the current state of the art of graphene manufacturing. Such a device must fit within a grain of graphene, nominally $10 \mu \mathrm{m} \times 10 \mu \mathrm{m}$. To ensure that the assumption of uniform circular motion is valid, arc lengths must be less than graphene's mean free path, nominally $\sim 1-2 \mu \mathrm{m}$ at $293 \mathrm{~K}$ at carrier concentrations of $10^{12} \mathrm{~cm}^{-2}$ [10] [23]. 


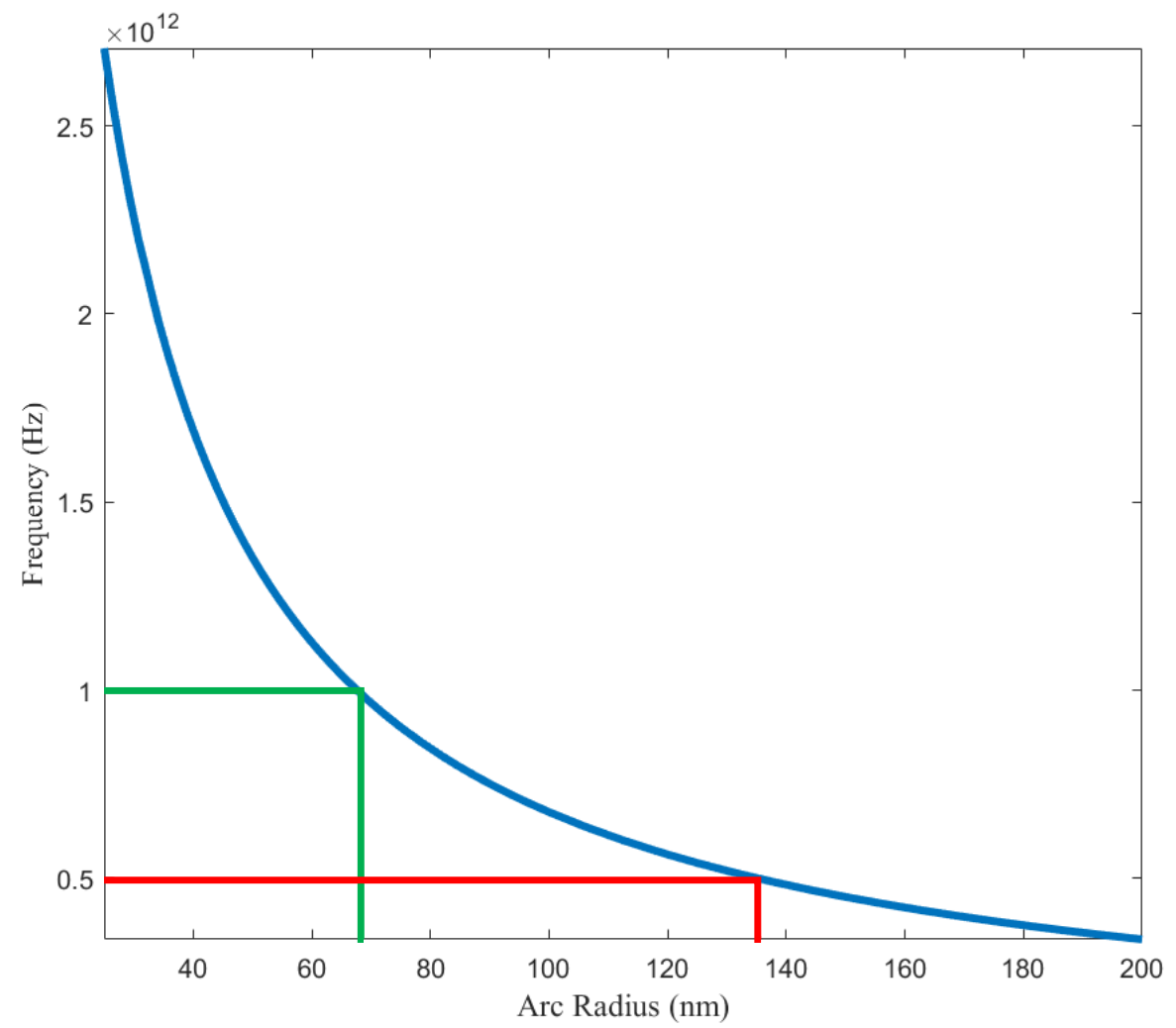

Figure 2 Trendline of emitted frequency vs. arc radius for a rotating dipole with a tangential velocity of $v_{\text {sat }}=4.25 \times 10^{7} \mathrm{~cm} / \mathrm{s}$. The target frequencies of $0.5 \mathrm{THz}$ and $1.0 \mathrm{THz}$ require an arc radius of $135 \mathrm{~nm}$ and $67 \mathrm{~nm}$ respectively.

For a rotating dipole, the solutions to Maxwell's equations yields the following radiated fields in spherical coordinates:

$$
\begin{aligned}
\vec{E}=\frac{\mu_{0} p_{0} \omega}{4 \pi r}\left\{\cos (\theta)\left[\cos \left(\omega\left(t-\frac{r}{c}\right)\right) \cos (\phi)+\sin \left(\omega\left(t-\frac{r}{c}\right)\right) \sin (\phi)\right] \hat{\theta}\right. \\
\left.-\left[\cos \left(\omega\left(t-\frac{r}{c}\right)\right) \sin (\phi)+\sin \left(\omega\left(t-\frac{r}{c}\right)\right) \cos (\phi)\right] \hat{\phi}\right\} \\
\vec{B}=\frac{\mu_{0} p_{0} \omega}{4 \pi r c}\left\{\left[\cos \left(\omega\left(t-\frac{r}{c}\right)\right) \sin (\phi)+\sin \left(\omega\left(t-\frac{r}{c}\right)\right) \cos (\phi)\right] \hat{\theta}\right. \\
\left.+\cos (\theta)\left[\cos \left(\omega\left(t-\frac{r}{c}\right)\right) \cos (\phi)+\sin \left(\omega\left(t-\frac{r}{c}\right)\right) \sin (\phi)\right] \hat{\phi}\right\}
\end{aligned}
$$

where $p_{0}$ is the dipole moment, $\left(p_{0}=q \cdot r_{\text {arc }}\right), \omega$ is the charge's angular velocity $(\omega=$ $\left.v_{\text {sat }} / r_{\text {arc }}\right), \mu_{0}$ is the vacuum permeability, $t$ is the time parameter; $r, \theta$, and $\phi$ are the respective radial, altitude, and azimuth coordinates with corresponding unit vectors $\hat{r}, \hat{\theta}$, and $\hat{\phi}$.

The concept of operation requires all the available charges in the graphene arc to be concentrated into a single point charge. Such a condition can at best be guaranteed for a time less than or equal to the mean scattering time in the substrate. Consequently, the arc length of the graphene arc should be less than the mean free path. For longer lengths, scattering mechanisms will adversely affect the uniform circular motion required in rotating dipole model. Furthermore, in a solid-state implementation, the uniform circular motion can only hold while charge carriers 
are in transit and not being injected or recombined at the source and drain terminals. In addition, the rotating dipole model has to be modified to accommodate the semicircular trajectory of the carriers - unlike the circular motion of the classical problem.

For simple cyclotron radiation, the circular geometry is the most common. To accommodate the source and drain terminals for the solid-state implementation, a semicircular arc is chosen as this guarantees that the source and drain are maximally separated by a distance equivalent to the circle's diameter. Such a design choice is preferable from a fabrication standpoint as both longer and shorter arc lengths would have sharp features that may not be reproduced correctly when fabricated. Additionally, the maximal separation reduces parasitic capacitances between the source and drain terminals. Having constrained the design to a semicircular arc, the transit time of the charge carriers is valid only for one half of an orbital period in the classic rotating dipole problem. To understand the behavior of this now modified problem, equations (2) and (3) are multiplied by a window function $[H(t)-H(t-T / 2)]$, where $H(t)$ is the Heaviside step function. A Fourier transform of the modified classical solution is calculated over the valid times of 0 to $\mathrm{T} / 2$ where $\mathrm{T}$ is the orbital period in the classic problem. It is anticipated that this modified problem will not radiate at a single frequency like the classic problem in which a Fourier transform yields a Dirac function at the angular velocity. Instead a spreading of the frequencies is expected about the angular velocity of the rotating dipole. If that is the case, then the shape of the spectrum would need to be determined in addition to the frequency that yields the most power. A dimensionless and reparametrized expression for the Fourier transformed fields is given below (4) where $x$ is the ratio of the frequency parameter $\omega$ to the charge's angular velocity $\omega_{0}$. Given the equivalence of the electric and magnetic fields by multiplication of an orthogonal unit vector and a factor of the speed of light, depending on the unit system - only the electric field is shown.

$\vec{E}(x, \phi, \theta)=\frac{\left(1+e^{-i \pi x}\right)(\cos \phi-i x \sin \phi) \hat{\phi}+\cos \theta\left(1+e^{-i \pi x}\right)(\cos \phi+i x \sin \phi) \hat{\theta}}{x^{2}-1}$

An expression for dimensionless Fourier transformed Poynting vector $\vec{S}=\vec{E} \times \vec{H}$ can be written as follows:

$\vec{S}=\frac{2(1+\cos (\pi x))\left[x^{2}\left(1-\cos ^{2} \phi \sin ^{2} \theta\right)+1-\sin ^{2} \phi \sin ^{2} \theta\right]}{\left(x^{2}-1\right)^{2}} \hat{r}$

From the dimensionless Poynting vector, critical values for this expression are sought to understand how the power output $S$ is related to normalized frequency $\omega / \omega_{0}$. A numerical evaluation of the Poynting vector expression yields a maximum at $x \approx 1.36$ (Figure 3Figure 2 ). The resulting pattern radiates in all directions, but is more biased along the $\mathrm{x}$-axis (Figure 4Figure 3). For $x=1$, the expression for the dimensionless Poynting vector yields the same value everywhere in the horizontal plane - a result identical to the unmodified problem. Lastly, for frequencies ranging from $0<x<1$ the classic dipole shape is recovered in which the lobes are oriented along the $\mathrm{x}$-axis and no radiation along the $\mathrm{y}$-axis. Another maximum is observed at $x \approx 3.6$, however there will not be a large contribution at this frequency or any other frequencies beyond the first peak at $x \approx 1.36$. 


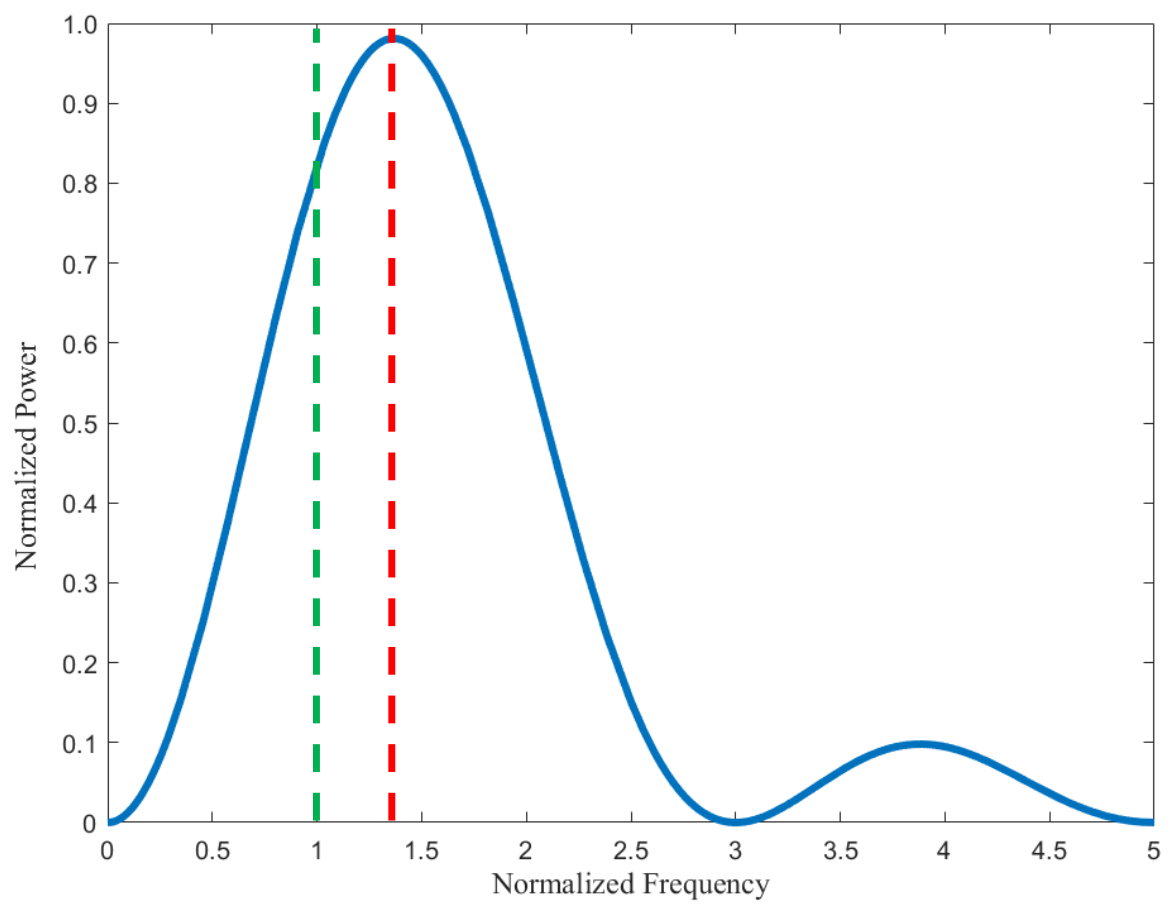

Figure 3 Calculated frequency spectrum for transient rotating dipole lasting $1 / 2$ of a full orbital period. The peak emission occurs at a normalized frequency at $\sim 1.36$. Another peak occurs at $\sim 3.6$.

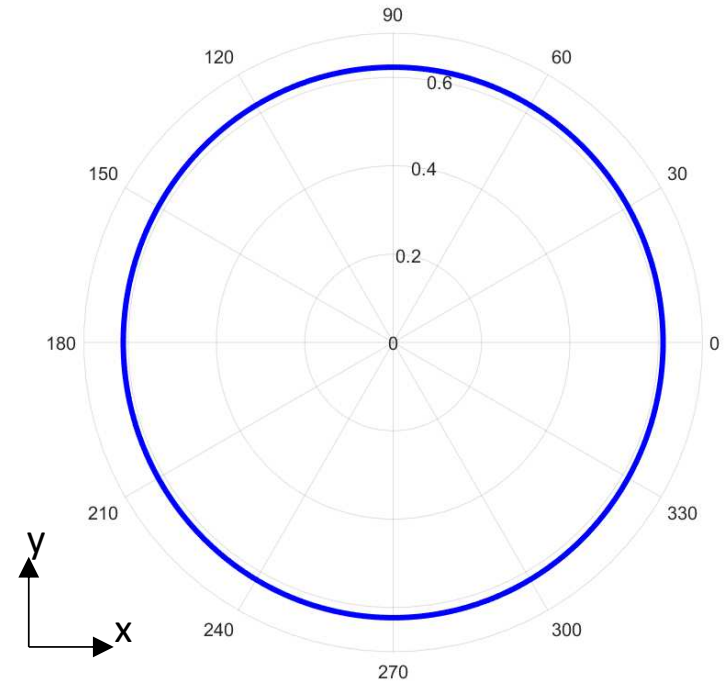

(a)

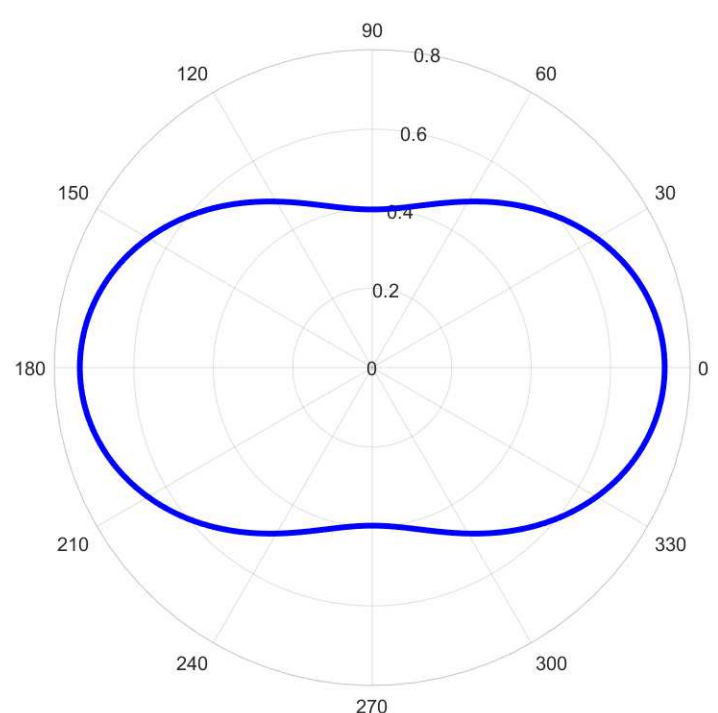

(b)

Figure 4 Calculated radiation patterns for transient rotating dipole lasting $1 / 2$ of a full orbital period (arbitrary scale) in the orbital plane. (a) Radiation pattern for normalized frequency equal to 1 . The circular pattern is identical to the steady state rotating dipole in which the emitted frequency and angular velocity are equivalent. Power is distributed uniformly in the plane of rotation at this frequency. (b) Radiation pattern for normalized frequency equal to $\sim 1.36$. Power is distributed in all directions of the orbital plane, but is more biased along the $\mathrm{x}$-axis. 


\section{Results}

\section{Dipole Model}

The simulations for the dipole model agree with the analytical model in that the target frequency is achieved. However, the peak power emission is at a frequency greater than the target. For the $0.5 \mathrm{THz}$ system, the peak power occurs at $0.67 \mathrm{THz}$ (Figure 5). For the $1 \mathrm{THz}$ system, the peak power occurs at $1.17 \mathrm{THz}$. Like the analytical studies, additional peaks at about $3 \mathrm{x}$ the target frequency are present. In terms of spatial distribution, the target frequency emits uniformly in the plane of rotation in agreement with the theoretical calculations (Figure 6). The peak frequency emits in all directions but is more biased along the $\mathrm{x}$-axis. From the steady state dipole simulations, the $0.5 \mathrm{THz}$ system emits a total power of $15.55 \mathrm{pW}$ and the $1 \mathrm{THz}$ system emits a total power of $25.4 \mathrm{pW}$. The simulated steady state dipole power emissions for both cases slightly overestimate the analytical results of $13.8 \mathrm{pW}$ for the $0.5 \mathrm{THz}$ system and $22.24 \mathrm{pW}$ for the $1 \mathrm{THz}$ system.

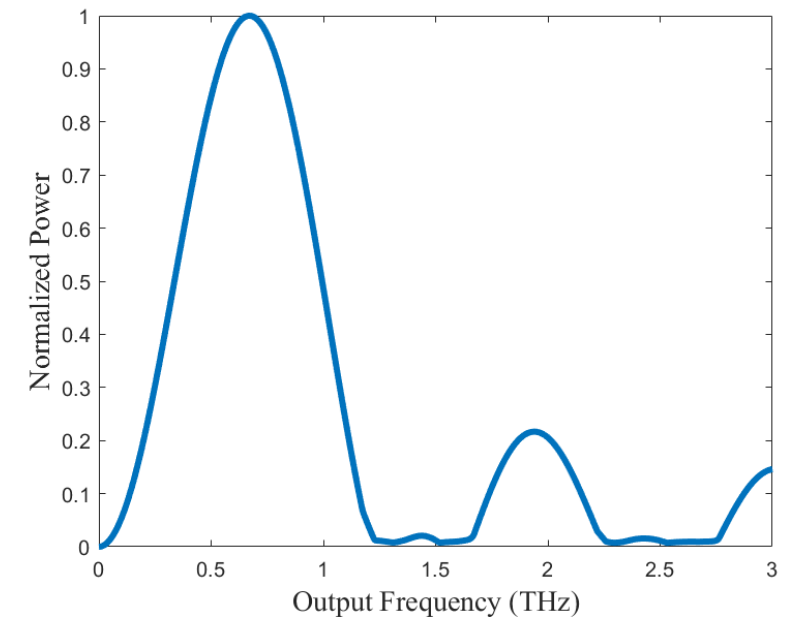

(a)

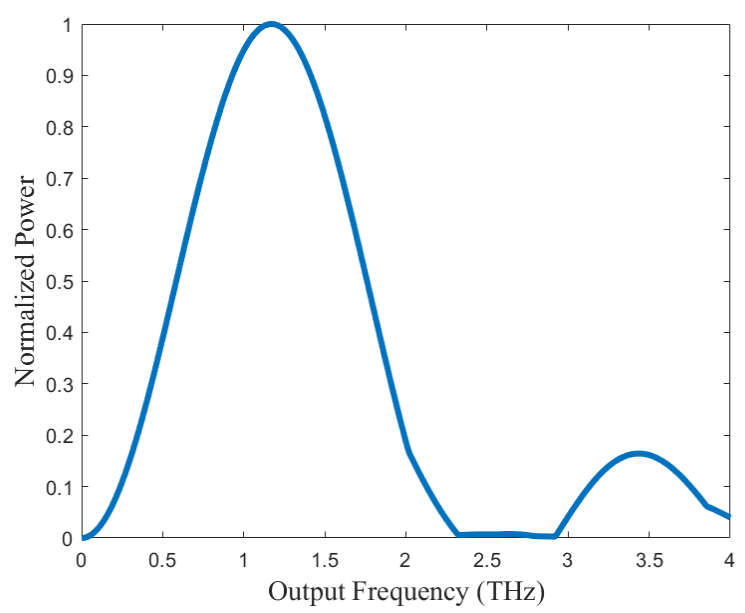

(b)

Figure 5 Simulated frequency spectra for the transient rotating dipole model. (a) Frequency spectrum of the simulated $0.5 \mathrm{THz}$ system. A peak emission is observed at $0.67 \mathrm{THz}$. (b) Frequency spectrum of the simulated $1 \mathrm{THz}$ system. A peak emission is observed at $1.17 \mathrm{THz}$ 


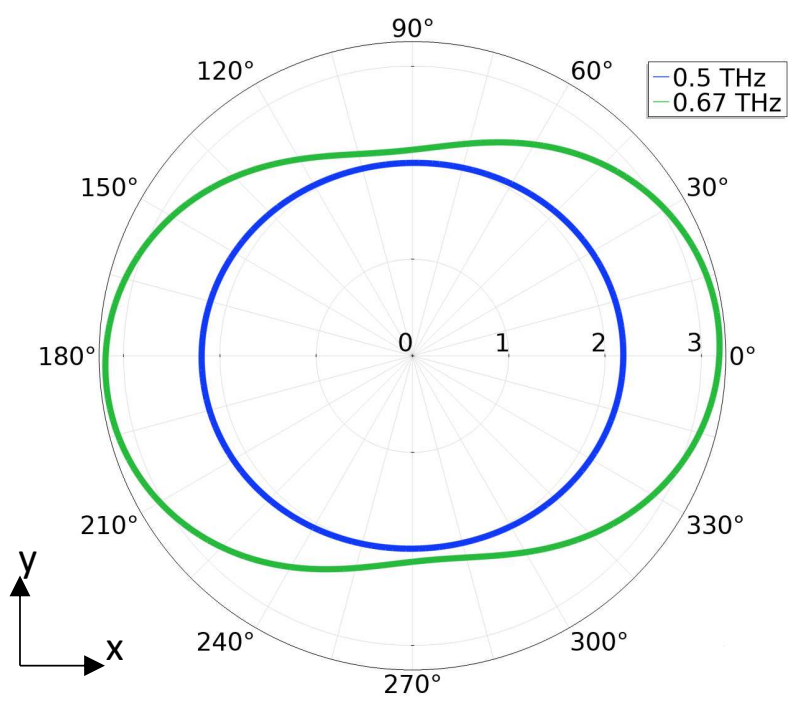

(a)

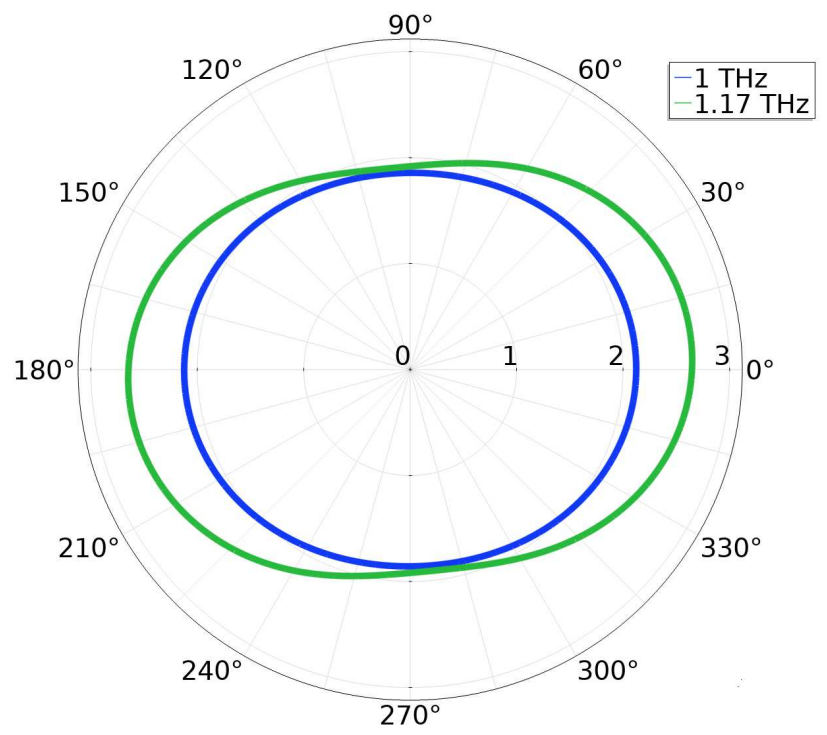

(b)

Figure 6 Simulated radiation patterns for the transient rotating dipole model (arbitrary scale) in the orbital plane. (a) Radiation pattern for the $0.5 \mathrm{THz}$ system. The design frequency of $0.5 \mathrm{THz}$ (blue) radiates uniformly in all directions in the plane of rotation. The peak frequency $0.67 \mathrm{THz}$ (green) radiates in all directions in the orbital plane, but is more biased along the x-axis. (b) Radiation pattern for the $1 \mathrm{THz}$ system. The design frequency of $1 \mathrm{THz}$ (blue) radiates uniformly in all directions in the plane of rotation. The peak frequency of $1.17 \mathrm{THz}$ radiates in all directions of the orbital plane, but is biased along the $\mathrm{x}$-axis.

\section{Arc Model}

The arc simulations were performed to determine for the effect of finite device width. For both designs, the target frequencies of $0.5 \mathrm{THz}$ and $1 \mathrm{THz}$ are achieved (Figure 7). For the 0.5 $\mathrm{THz}$ design, the peak power is emitted at $0.57 \mathrm{THz}$, while the $1 \mathrm{THz}$ design's peak power is emitted at $1.33 \mathrm{THz}$. Total power is calculated to be $18.4 \mathrm{pW}$ for the $0.5 \mathrm{THz}$ arc and $13.8 \mathrm{pW}$ for the $1 \mathrm{THz}$ arc. Due to limitations in COMSOL's capabilities, the radiation patterns for the arcs did not capture the particle dynamics of the charge carriers in the arc (Figure 8). As a result, the expected radiation pattern from a rotating charge is not produced by the simulations. The shape instead resembles that of a dipole oscillating along the $\mathrm{x}$-axis.

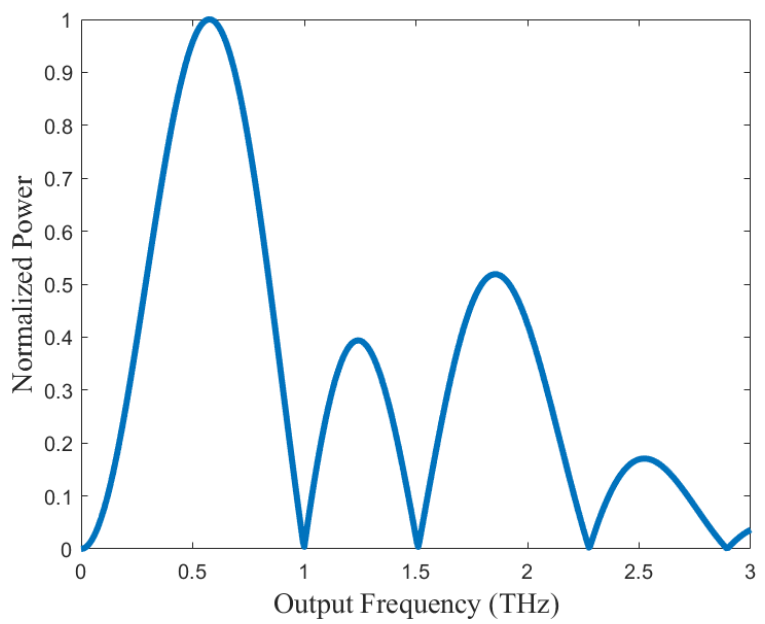

(a)

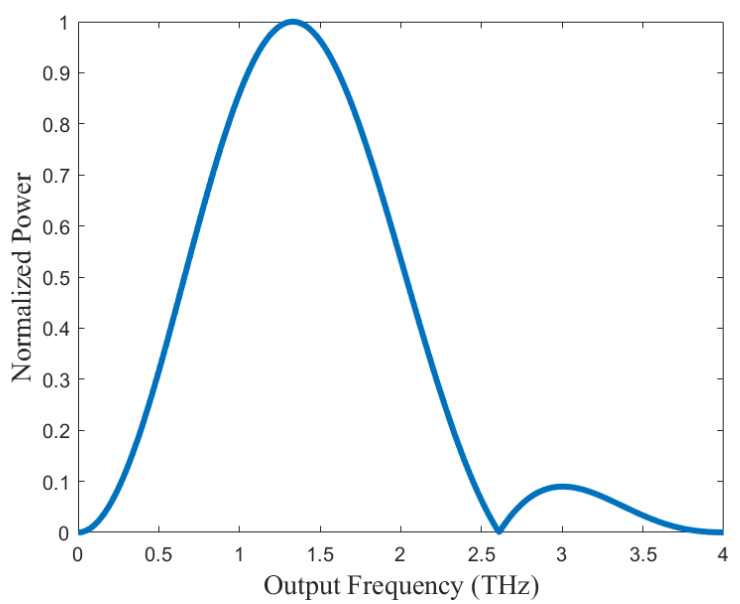

(b) 
Figure 7 Simulated frequency spectra for the transient arc model. (a) Frequency spectrum of the $0.5 \mathrm{THz}$ system. A peak occurs at $0.57 \mathrm{THz}$ with secondary peak occurring at $1.8 \mathrm{THz}$. A tertiary peak occurs at $1.25 \mathrm{THz}$, previously not predicted by the dipole model. (b) Frequency spectrum of the $1 \mathrm{THZ}$ system. A peak occurs at $1.33 \mathrm{THz}$ with a secondary peak at $3 \mathrm{THz}$.

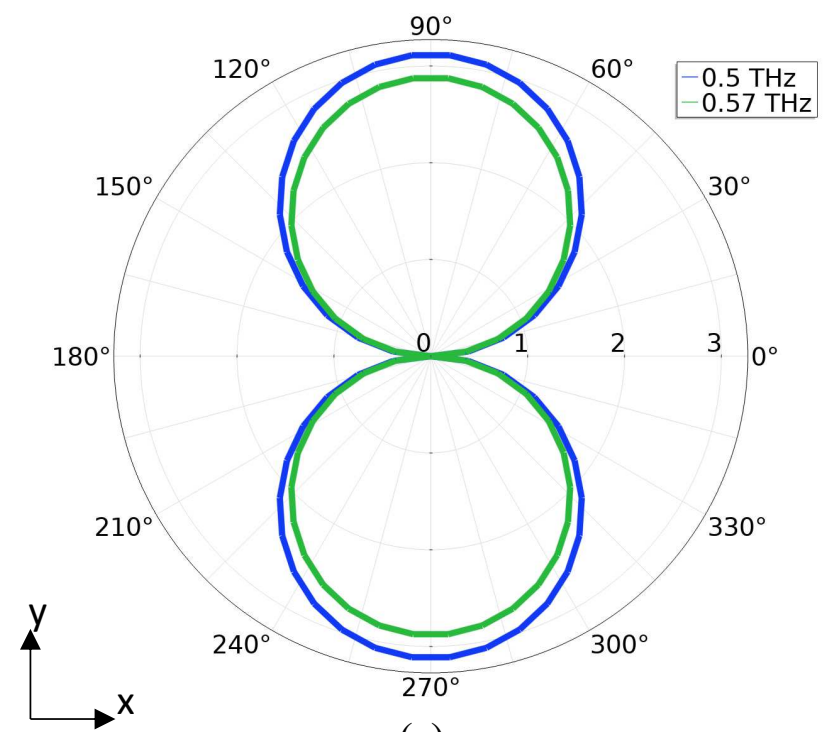

(a)

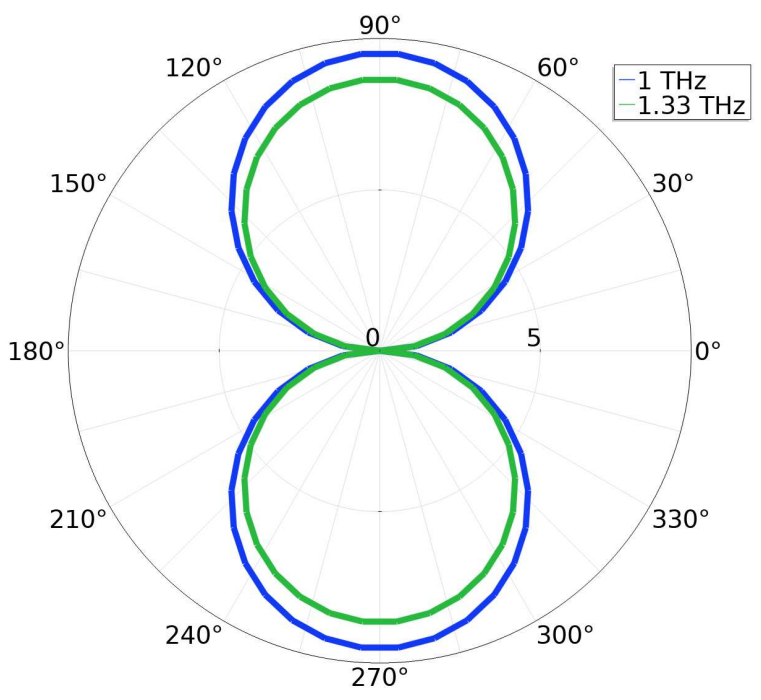

(b)

Figure 8 Simulated radiation patterns for the transient arc models in the orbital plane (arbitrary scale). (a) The $0.5 \mathrm{THz}$ system and the $1 \mathrm{THz}$ system (b) at the design frequency (blue) and at peak emission (green). The finite element software used does not account for particle dynamics and hence does not produce the expected circular rotating dipole radiation pattern. The shape instead resembles a dipole oscillating along the $\mathrm{x}$-axis.

To illustrate the deviation from the point charge models, DC simulations were performed on the respective arcs (Figure 9). The simulations with an applied bias of 1V indicate that current flows from the source, on the right hand side, to the drain, on the left, along the field gradient. The current is most closely concentrated towards the inner radius as indicated by arrow length and thickness. Unlike the point charge assumption, in the arc, charges are distributed along the width of the device.

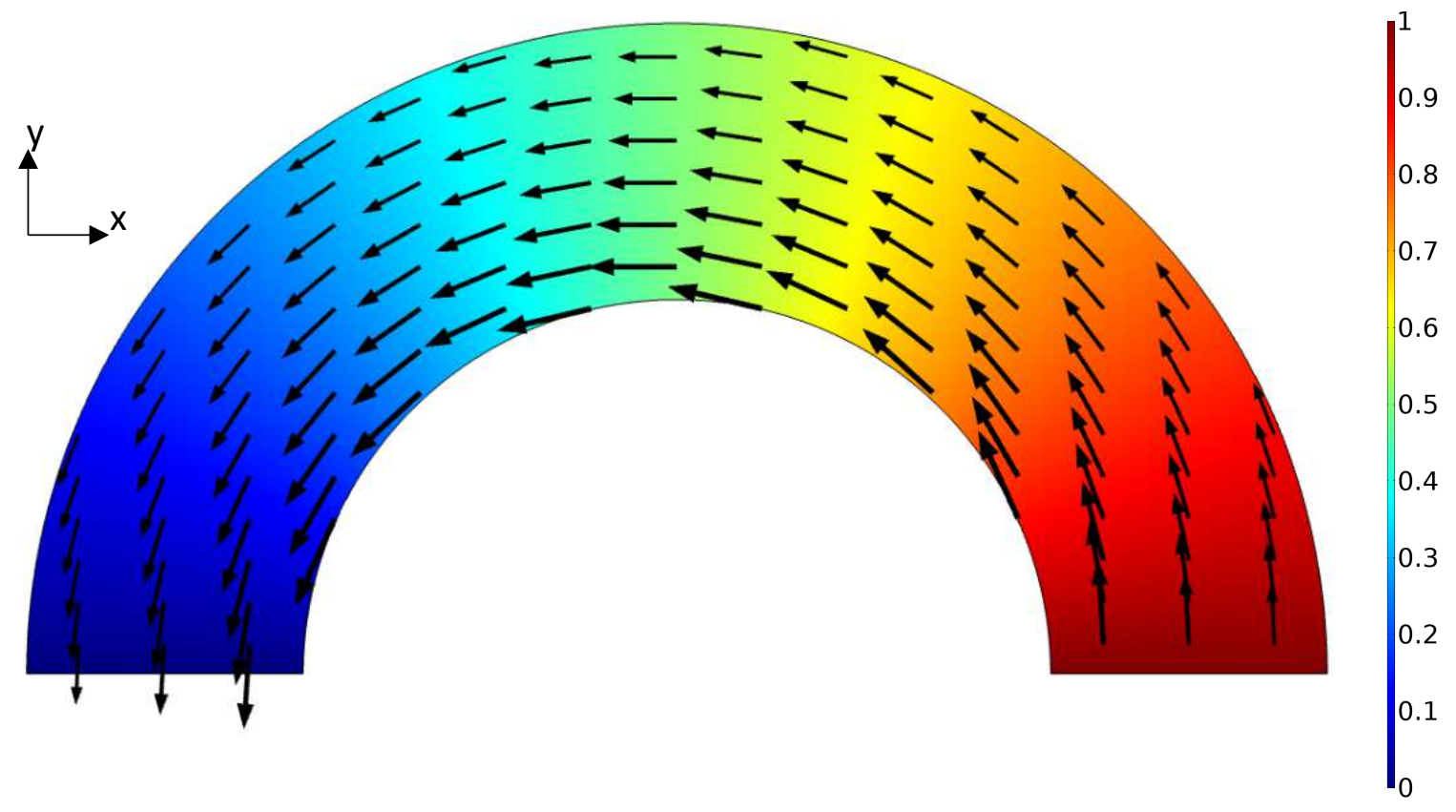


Figure 9 Simulated current density under DC bias of $1 \mathrm{~V}$ potential difference between source and drain. In both systems, current flows along the arc with the highest current density at the inner radius as indicated by arrow size. This is unlike the point charge assumption in which all of the charge located at the inner radius.

Another deviation from the point charge model pertains to device stimulation. For the concept of operation, a transient pulse with a voltage amplitude of $1 \mathrm{~V}$ is applied between source and drain terminals. A $40 \mathrm{GHz}$ Gaussian pulse was used in the preceding simulations as it is a sufficiently high frequency that is still attainable with existing technology. With lower frequency stimuli, the frequency spectrum is expected to be degraded with more spurious components being present. The emission spectrum was obtained for additional stimuli at $4 \mathrm{GHz}$ and $10 \mathrm{GHz}$. Figure 10 shows the spectral response of the $0.5 \mathrm{THz}$ system. Remarkably, the spectrum shape is consistent over all the stimulus frequencies.

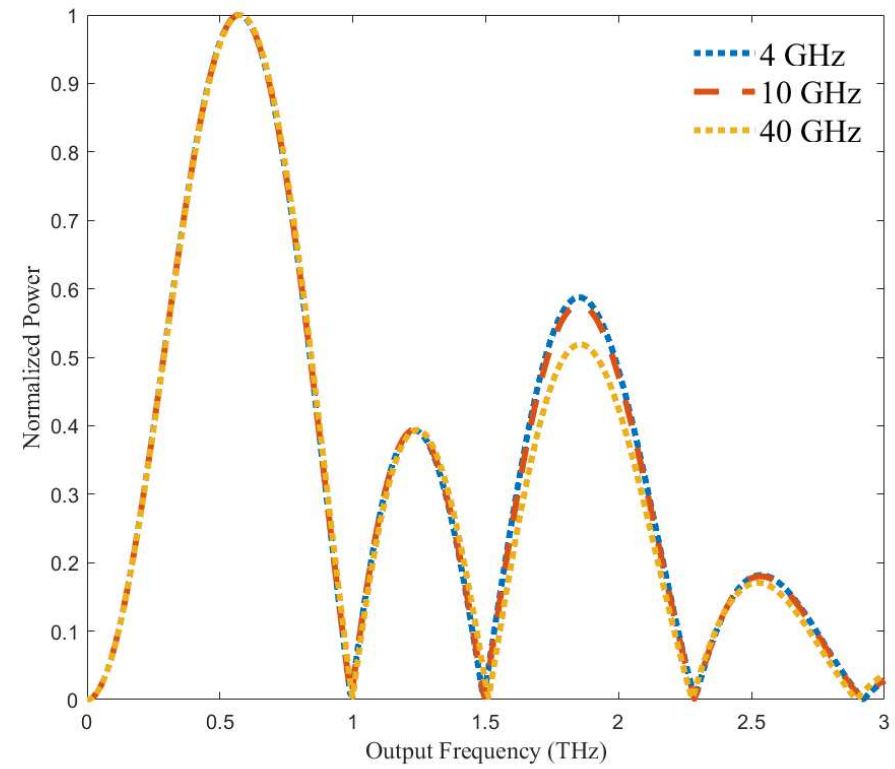

Figure 10 Effect of stimulus frequency on emission spectrum with $4 \mathrm{GHz}, 10 \mathrm{GHz}$, and $40 \mathrm{GHz}$ stimuli. Normalized spectra over the set of applied stimuli for the $0.5 \mathrm{THz}$ design. For all stimuli, the shape is consistent. The $4 \mathrm{GHz}$ and $10 \mathrm{GHz}$ slightly overshoot the $40 \mathrm{GHz}$ secondary peak at $1.8 \mathrm{THz}$.

The simulation results over a set of different stimulus frequencies [Figure 10] indicate that the emission spectrum is independent of the stimulus frequency. This result was not expected as it is anticipated that the device would be less responsive at frequencies farther away from the target frequency and thus the emission spectrum would more likely consist of spurious emissions. The device's emission spectrum is a function of its geometry defined by the arc's inner radius.

\section{Discussion}

The possibility of terahertz emission from a solid-state cyclotron-radiation emitter device with a graphene substrate has been demonstrated. A simplified an analytical model showed that the device can first be modeled as the classic rotating dipole problem by assuming that all the substrate charges can be treated as a single point charge. Two finite element models were also created to first, verify the analytical results and second, account for the finite dimensions of the graphene arcs. Both output power and spectral characteristics were obtained by all three models. Table 1 shows the calculated output power. For the $0.5 \mathrm{THz}$ system all calculations agree within the same order of magnitude and are very close. For the $1 \mathrm{THz}$ system, the simulated dipole 
agrees with the Larmor calculation while the simulated arc underestimates by nearly $10 \mathrm{pW}$. Given the results of both systems, a single graphene solid state cyclotron operating at 0.5 to 1.0 $\mathrm{THz}$ is expected to produce on the order of $10 \mathrm{pW}$ of total radiated power.

\begin{tabular}{|c|c|c|}
\hline \multirow{2}{*}{ Model } & \multicolumn{2}{|c|}{ Power (pW) } \\
\cline { 2 - 3 } & $\mathbf{0 . 5} \mathbf{~ T H z}$ & $\mathbf{1 ~ T H z}$ \\
\hline Analytical & 13.8 & 22.0 \\
\hline FE Dipole & 15.5 & 25.4 \\
\hline FE Arc & 18.4 & 13.8 \\
\hline
\end{tabular}

Table 1 Output power of the graphene nanocyclotrons estimated using 3 different models.

In terms of radiated frequency spectrum, the transient operating nature of this device implies that even with the point charge assumption that is constrained to orbit a fixed radius, the emission spectrum would be distributed around the angular velocity. This distribution is expected to contain a frequency equivalent to the angular velocity of the orbiting charge with a peak power emission at a frequency $\sim 1.36$ times the angular velocity. For the $0.5 \mathrm{THz}$ system both the simulated dipole and the simulated arc produce the target frequency of $0.5 \mathrm{THz}$ and their peak emissions occur at $0.67 \mathrm{THz}$ and $0.57 \mathrm{THz}$ respectively. This gives a ratio of 1.34 for the simulated dipole and 1.14 for the simulated arc. For the $0.5 \mathrm{THz}$ system, the simulated dipole approaches the theoretically predicted peak while the simulated arc falls slightly short. The 1 $\mathrm{THz}$ system, however, yields a ratio of 1.17 for the simulated dipole and 1.33 for the simulated arc. For both systems, each of their respective simulations slightly underestimate the predicted peak frequency. It was expected that the simulated dipoles would yield the closest values since these approximations are equivalent to the analytical calculations. Given these results, one can expect the peak frequency to be higher than the target frequency between 1.14 and 1.36 times the angular velocity.

With the aforementioned result, one can design a system in which the desired frequency is the peak frequency and not the orbital angular frequency. Such a system could then achieve the same frequency emission with a larger physical footprint than a system in which the angular velocity is the target frequency. This would be advantageous from a prototyping fabrication perspective as the larger footprint would provide additional margin for the processing steps hopefully yielding high fidelity patterns over a design with smaller margins. It is important to mention that, designing for the peak emission will come at the expense of the uniform radiation pattern (Figure 6).

The predicted device power per area is on the order of $1 \mathrm{nW} / \mathrm{cm}^{2}$. This device performance is in the upper range of existing work on graphene wigglers which are expected to emit $1 \mathrm{pW} / \mathrm{cm}^{2}$ to $10 \mathrm{nW} / \mathrm{cm}^{2}$ [13]. Such power can be realized with large scale repetition using existing semiconductor processing methods. Given the geometric constraints of a semicircle, it is possible that the desired radiation pattern can be preserved over an array of these devices by having alternating oriented semicircles such that the source and drain terminals also alternate instead of a simple repetition and translation of the semicircle and associated interconnects. Such an implementation would piecewise form a full circle and would save on manufacturing space by reusing a terminal that can be used by two neighboring units.

The results of these simulations are promising enough to fabricate the proposed device. Further simulation work to be performed would focus on increasing device density and associated effects on device performance, namely the effects of the interconnect network on parasitic capacitance. 
A solid-state implementation of a $\mathrm{THz}$ cyclotron radiation source in graphene has been investigated through analytical dipole models and finite element simulation and results indicate that such a device is feasible. Designs in the $0.5 \mathrm{THz}$ to $1.5 \mathrm{THz}$ are supported by a semicircular arc geometry and can emit power in the range of $1 \mathrm{nW} / \mathrm{cm}^{2}$. With a highly scalable planar semicircular geometry along with existing semiconductor processing methods, this predicted power range can be realized and potentially leading to low cost $\mathrm{THz}$ emitter sources. 


\section{Methods}

Finite element simulations of the simple dipole model in both steady state and transient conditions followed by a semicircular arc of graphene in steady state and transient conditions were also performed. In COMSOL Multiphysics, a point dipole located in the center of the coordinates was created with charge equivalent to the sum of all free carriers of the graphene arcs that correspond to each target frequency $(0.5 \mathrm{THz}$ and $1 \mathrm{THz})$.

This value is obtained by multiplying the arc area by the charge density. In this study, a charge density of $10^{12} \mathrm{~cm}^{-2}$ is used as such a value can be realistically obtained at room temperature while having mean free paths on the order of $1 \mu \mathrm{m}$. For the $0.5 \mathrm{THz}$ system, the effective point charge corresponds to 1164 electrons while the effective point charge for the 1 THz system corresponds to 739 electrons. Using the RF module in COMSOL, the charge starts at a position $\vec{r}(r, \phi, \theta)=\left(r_{a r c}, 0, \frac{\pi}{2}\right)$ and orbits the origin in a counterclockwise manner. In the steady state condition, the charge is allowed to make complete orbits like in the classic rotating dipole problem. A scattering boundary condition was defined for a $10 \mu \mathrm{m}$ radius sphere containing the dipole. This model was used to calculate the maximum power output and is compared to the Larmor power formulations.

In addition, a transient dipole model was developed to capture the frequency spectrum and radiation pattern. Much like the steady state model, all the arc's charge is concentrated to a point and orbits the origin in a counterclockwise fashion at a fixed radius. Unlike the steady state model, the transient model is only valid for $1 / 2$ of an orbital period. The system is expected to emit a range of frequencies in which the target frequency is emitted uniformly in the plane of rotation. According to the analytical results (Figure 3Figure 2) a peak emission at a frequency $36 \%$ higher than the target frequency is also expected.

More realistic models were developed to help prove the concept, where a finite arc width $(100 \mathrm{~nm})$ is considered. The graphene layer was modeled as a boundary with sheet resistivity specified by the manufacturer $430 \Omega$ /sq [17]. First, using COMSOL's AC/DC module, a direct current (DC) simulation was performed. The straight edges of the arc were set as ports with fixed potential where one was ground and the other $1 \mathrm{~V}$. This simulation allows for verification of the field gradient and current flow along the arc. Next, a steady state simulation of the arcs stimulated at their respective target frequencies is performed to calculate the power output. Lastly, a transient simulation is performed on the arcs for a time of $1 / 2$ of a full orbital period with a Gaussian pulse stimulus centered at $40 \mathrm{GHz}, 10 \mathrm{GHz}$, and $4 \mathrm{GHz}$. This stimulus frequencies were chosen as they can be readily obtained with existing technology. 


\section{References}

[1] Novoselov, K. S., Jiang, D., Schedin, F., Booth, T.J., Khotkevich, J.J., Morozov, S.V. \& Geim, A.K. Two-dimensional atomic crystals. Proceedings of the National Academy of Sciences. 102, 10451-10453 (2005).

[2] Ferrari, A. C. et al., Raman spectrum of graphene and graphene Layers. Phys. Rev. Letters. 97, 187401-187404 (2006).

[3] Novoselov, K. S. Electric field effect in atomically thin carbon films. Science. 306, 666669 (2004).

[4] Geim, A.K. \& MacDonald, A. H., Graphene: Exploring carbon flatland. Phys. Today, 60, 35-41, (2007).

[5] K. S. Novoselov et al., Two-dimensional gas of massless Dirac fermions in graphene. Nature. 438, 197-200 (2005).

[6] Lemme, M. C., Echtermeyer, T. J., Baus, M. \& Kurz, H. A graphene field-effect device. IEEE Electron Device Letters. 28, 282-284 (2007).

[7] Nikitin, A.Y, Guinea, F., García-Vidal, F. J. \& Martín-Moreno, L. Edge and waveguide terahertz surface plasmon modes in graphene microribbons. Phys. Rev. B. 84, 161407161411 (2011).

[8] Perruisseau-Carrier, J. Graphene for antenna applications: Opportunities and challenges from microwaves to THz. Loughborough Antennas \& Propagation Conference (LAPC), 2012.

[9] Yao, Y. et. al. Broad Electrical Tuning of Graphene-Loaded Plasmonic Antennas. Nano Letters. 13, 1257-1264, (2013).

[10] Tse, W.K., Hwang, E. H., \& Das Sarma, S. Ballistic hot electron transport in graphene. Appl. Phys. Letters. 93, 023128; 10.1063/1.2956669 (2008).

[11] Shishir, R. S. \& Ferry, D. K. Velocity saturation in intrinsic graphene. J. Phys: Condens. Matter. 21, 344201; 10.1088/0953-8984/21/34/344201 (2009).

[12] Tantiwanichapan, K., Wang, X., Swan, A. K. \& Paiella, R. Graphene on nanoscale gratings: a novel materials platform for $\mathrm{THz}$ electron-beam radiation. Adv. Photonics 2015: OSA Tech. Digest. https://doi.org/10.1364/IPRSN.2015.IM4A.2 (2015).

[13] Anwar, F., Carlos, C. R., Saraswat, V., Mangu, V. S., Arnold, M. S., \& Cavallo, F. Nanoscale graphene/Ge wigglers as building blocks for THz sources. AIP Adv. 7, 115015; $10.1063 / 1.4986513$ (2017).

[14] Cole, N. \& Antonsen. Jr., T. M., Cyclotron resonance gain for FIR and THz radiation in graphene. https://arxiv.org/abs/1602.08397 (2017).

[15] Jago, R., Winzer, T., Knorr, A. \& Malic, E. Graphene as gain medium for broadband lasers. Phys. Rev. B. 92, $085407-085413$ (2015).

[16] Morimoto, T., Hatsugai, Y., \& Aoki, H. Cyclotron radiation and emission in graphene. Phys. Rev. B. 78, 073406 - 073409 (2008).

[17] CheapTubes Inc. CVD graphene on $\mathrm{Si}-\mathrm{SiO} 24$ inch. https://www.cheaptubes.com/product/cvd-graphene-si-sio2-4inch (2020) 
[18] Gardner, C. L., Numerical simulation of a steady-state electron shock wave in a submicrometer semiconductor device. IEEE Transactions on Electron Devices. 38, 392398 (1991).

[19] Kunz, C. Synchrotron radiation. Meeting on Technology arising from High-energy Physics. http://cds.cern.ch/record/880472 (1974).

[20] Griffiths, D. J. :Dipole Raditation in Introduction to Electrodynamics: Fourth Edition. 482 - 488 (Pearson, 2013).

[21] Lemme, M. C. et al. Etching of graphene devices with a Helium ion beam. ACS Nano. 3, 2674-2676 (2009).

[22] Han, M. Y., Özyilmaz, B., Zhang, Y. \& Kim, P. Energy band-gap engineering of graphene nanoribbons. Phys. Rev. Letters. 98, 206805- 206808 (2007).

[23] Chen, J. H., Jang, C., Xiao, S., Ishigami, M., \& Fuhrer, M. S., Intrinsic and extrinsic performance limits of graphene devices on SiO2. Nature Nanotech. 3, 206-209 (2008).

Acknowledgements

The authors would like to thank Peter Crooker for proofreading and editing the manuscript.

\section{Author Contributions}

JP wrote the main manuscript text and prepared all figures. FA interpreted the figures and data. All authors reviewed the manuscript.

\section{Competing Interests}

The authors declare no competing interests. 


\section{Figures}

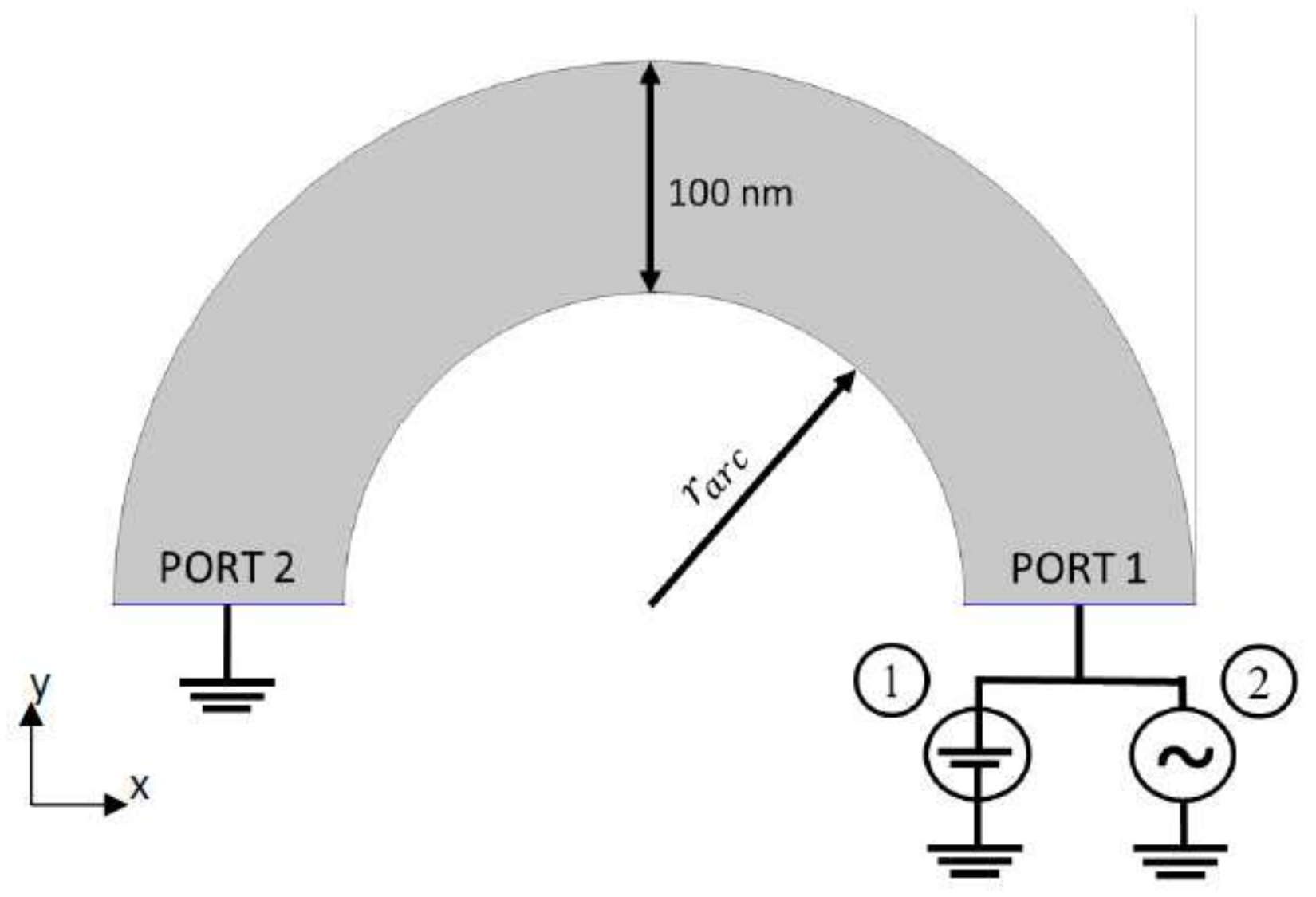

Figure 1

Schematic setup of the graphene arcs. In the dipole model, all of the arc's charges are concentrated at a single point and traverse a semicircular trajectory at a radius rarc. The motion of the charges is expected to produce cyclotron radiation at frequencies inversely proportional to rarc. For the finite element DC simulation (1), a constant $1 \mathrm{~V}$ was applied. For the finite element RF simulation (2), signals at $4 \mathrm{GHz}, 10$ $\mathrm{GHz}$, and $40 \mathrm{GHz}$ of $1 \mathrm{~V}$ amplitude were applied to port 1. 


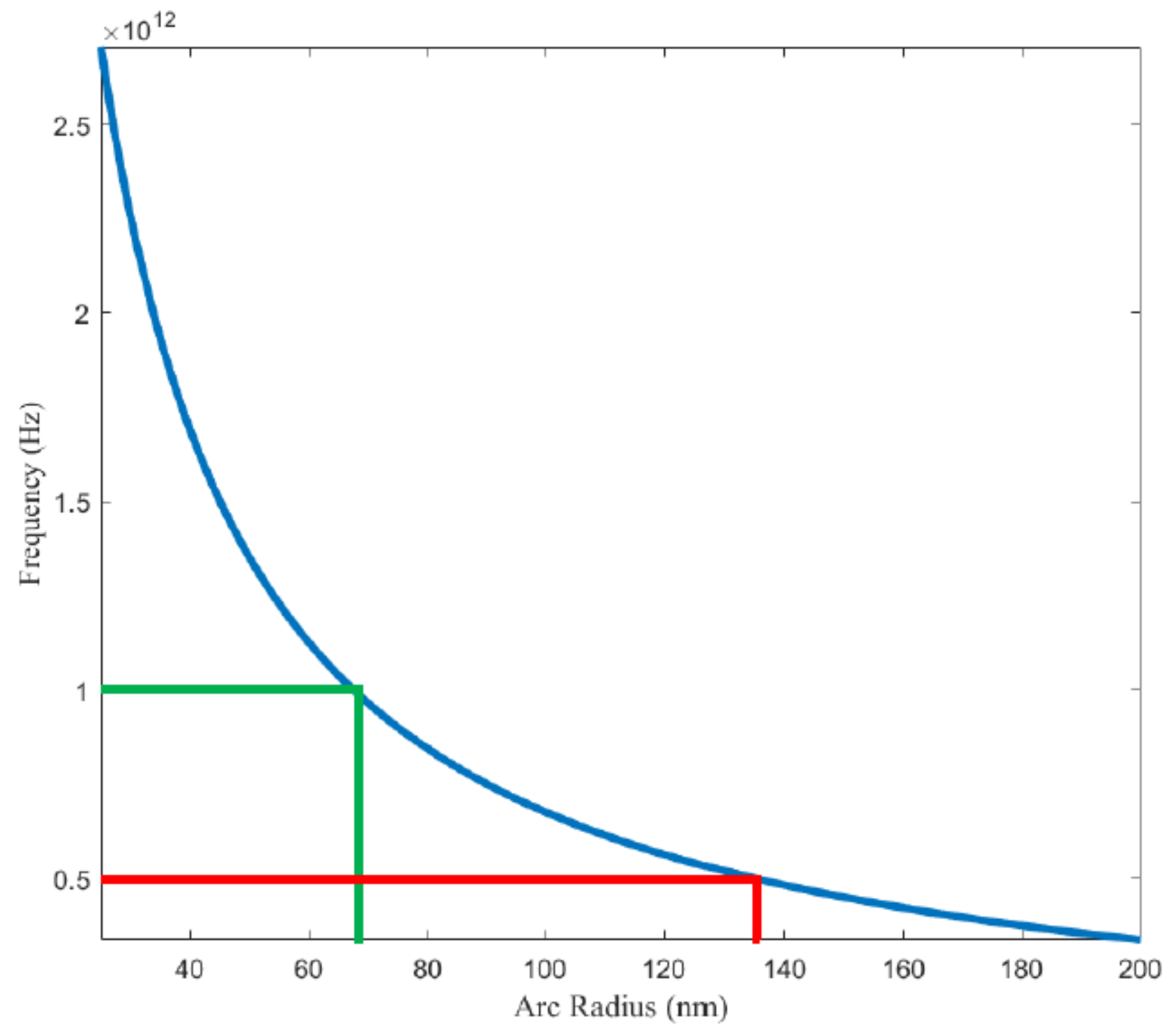

Figure 2

See manuscript for full figure caption. 


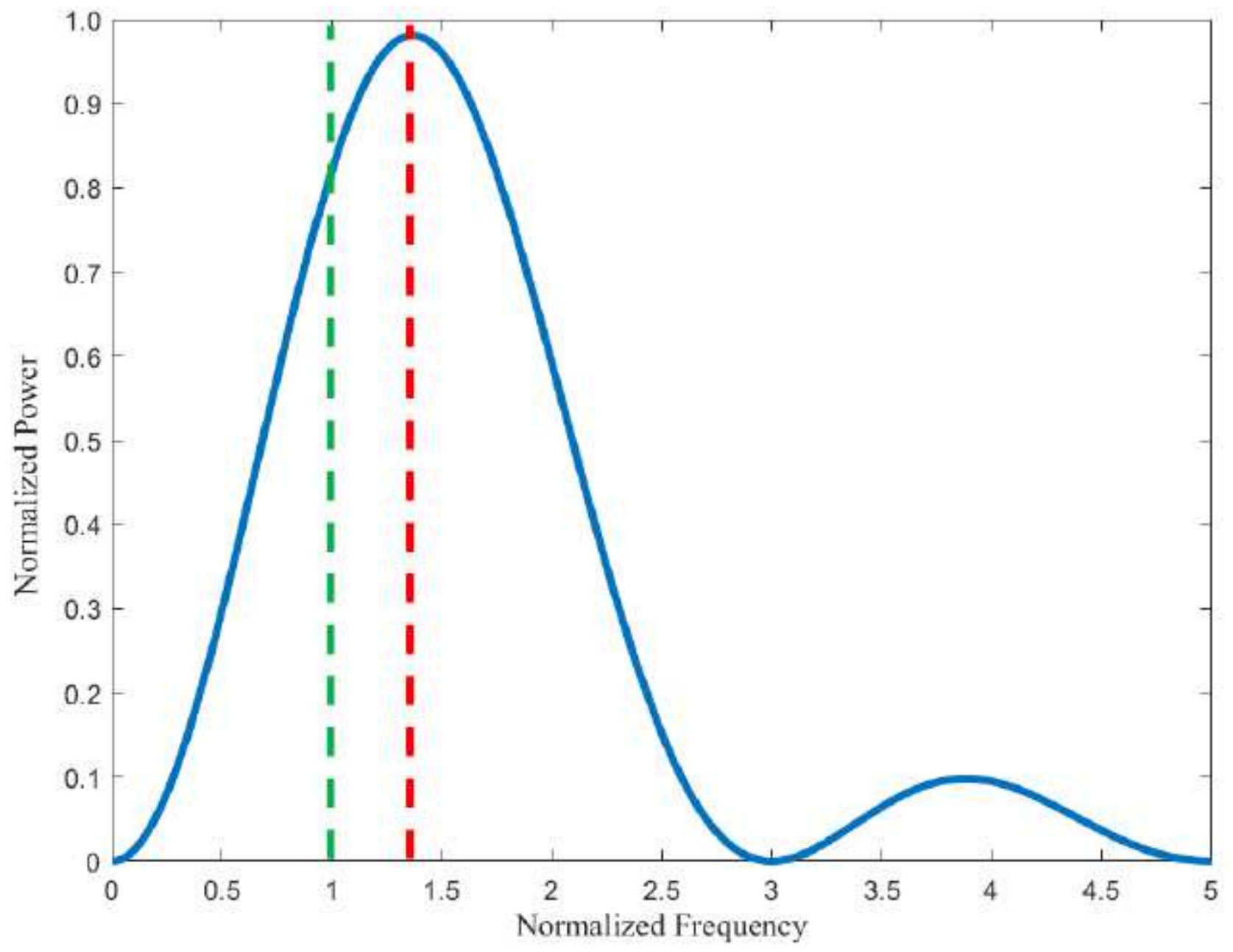

Figure 3

Calculated frequency spectrum for transient rotating dipole lasting $1 / 2$ of a full orbital period. The peak emission occurs at a normalized frequency at $~ 1.36$. Another peak occurs at $~ 3.6$. 


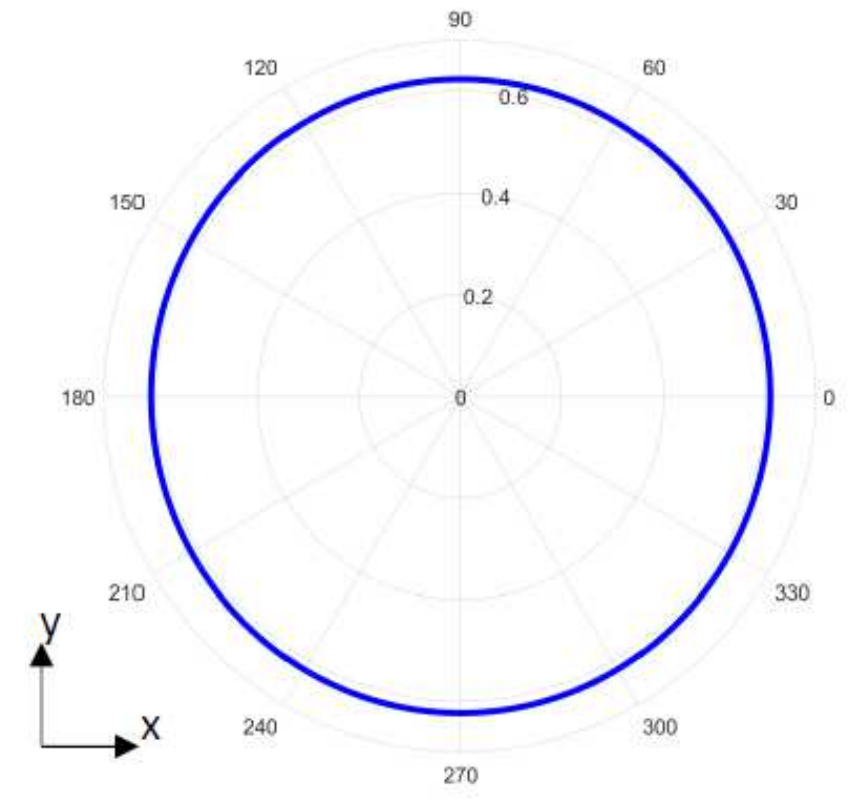

(a)

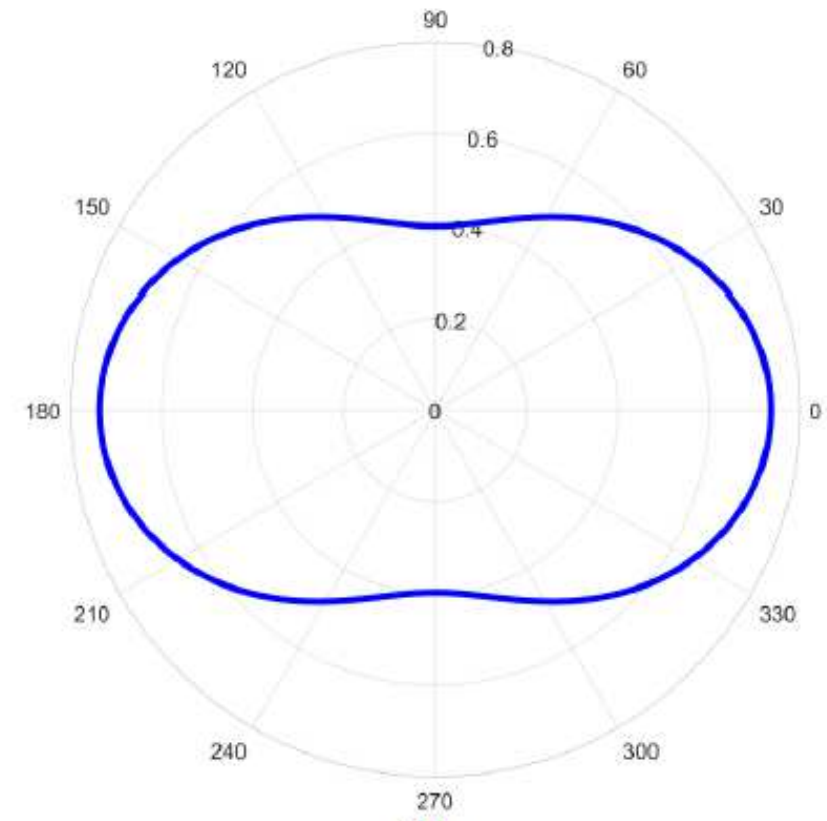

(b)

\section{Figure 4}

Calculated radiation patterns for transient rotating dipole lasting $1 / 2$ of a full orbital period (arbitrary scale) in the orbital plane. (a) Radiation pattern for normalized frequency equal to 1 . The circular pattern is identical to the steady state rotating dipole in which the emitted frequency and angular velocity are equivalent. Power is distributed uniformly in the plane of rotation at this frequency. (b) Radiation pattern for normalized frequency equal to $\sim 1.36$. Power is distributed in all directions of the orbital plane, but is more biased along the $x$-axis.

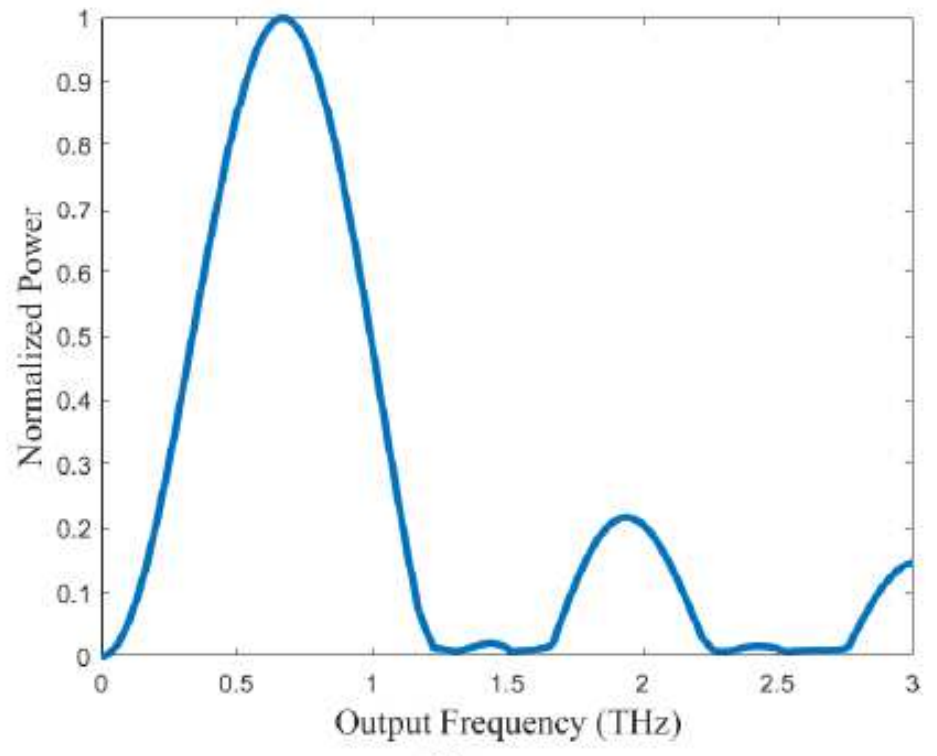

(a)

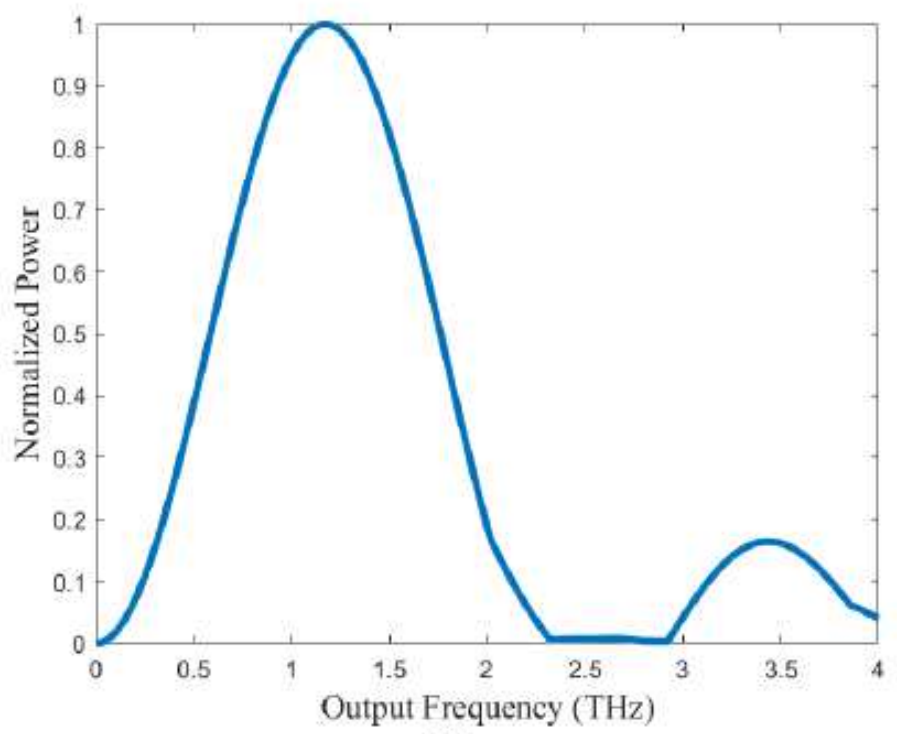

(b)

Figure 5 
Simulated frequency spectra for the transient rotating dipole model. (a) Frequency spectrum of the simulated $0.5 \mathrm{THz}$ system. A peak emission is observed at $0.67 \mathrm{THz}$. (b) Frequency spectrum of the simulated $1 \mathrm{THz}$ system. A peak emission is observed at $1.17 \mathrm{THz}$

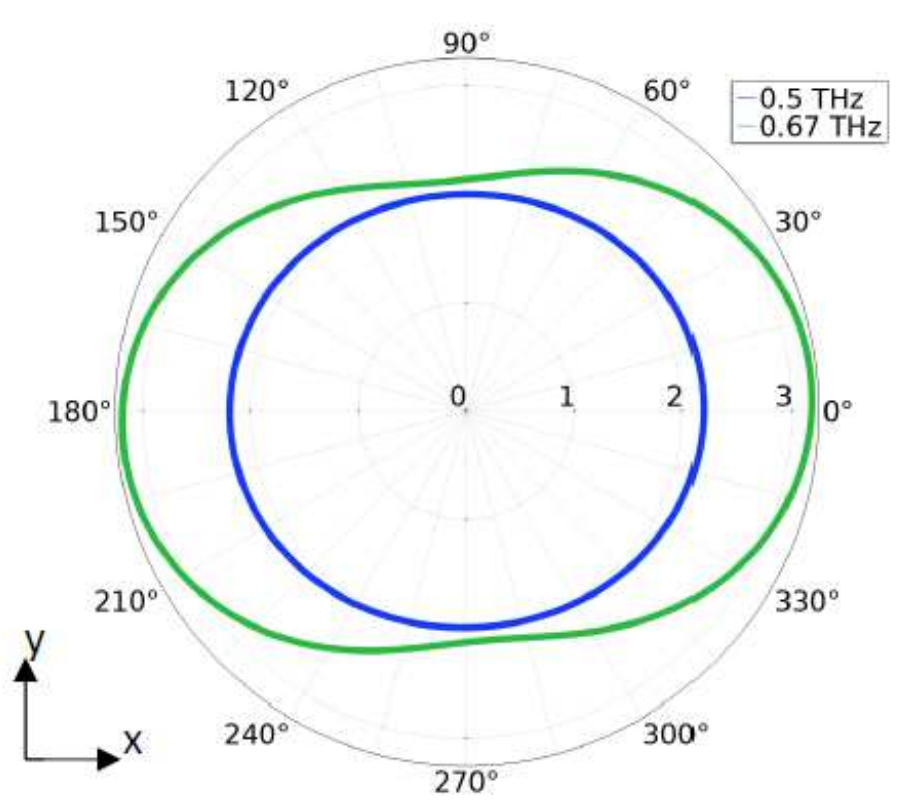

(a)

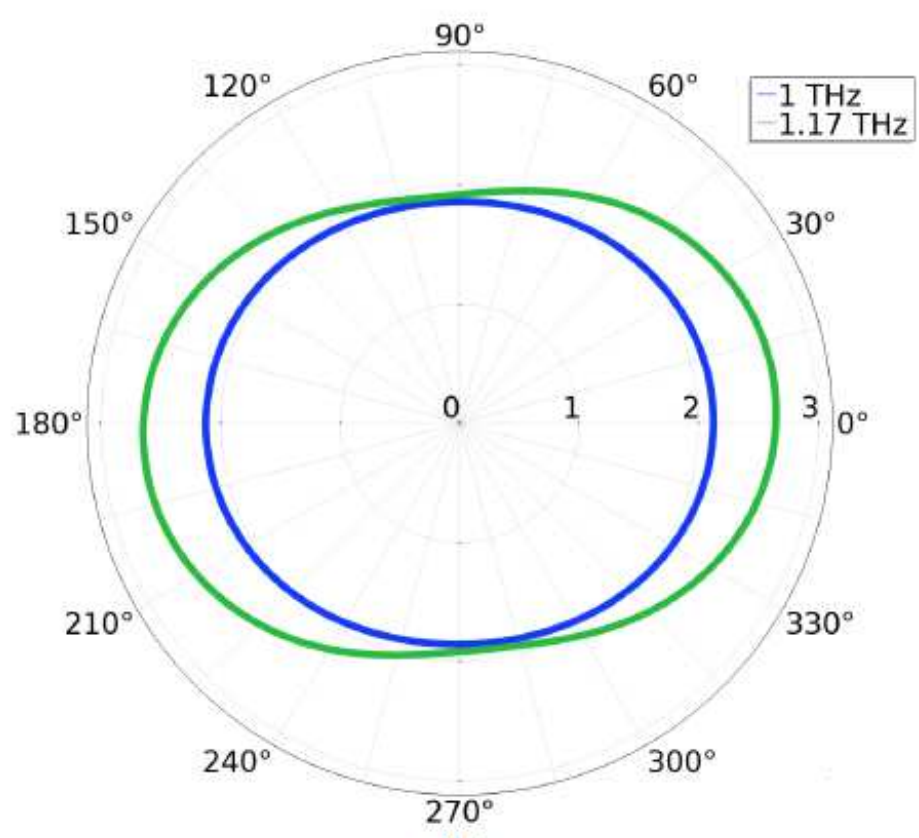

(b)

Figure 6

the $0.5 \mathrm{THz}$ system. The design frequency of $0.5 \mathrm{THz}$ (blue) radiates uniformly in all directions in the plane of rotation. The peak frequency $0.67 \mathrm{THz}$ (green) radiates in all directions in the orbital plane, but is more biased along the x-axis. (b) Radiation pattern for the $1 \mathrm{THz}$ system. The design frequency of $1 \mathrm{THz}$ (blue) radiates uniformly in all directions in the plane of rotation. The peak frequency of $1.17 \mathrm{THz}$ radiates in all directions of the orbital plane, but is biased along the $x$-axis.

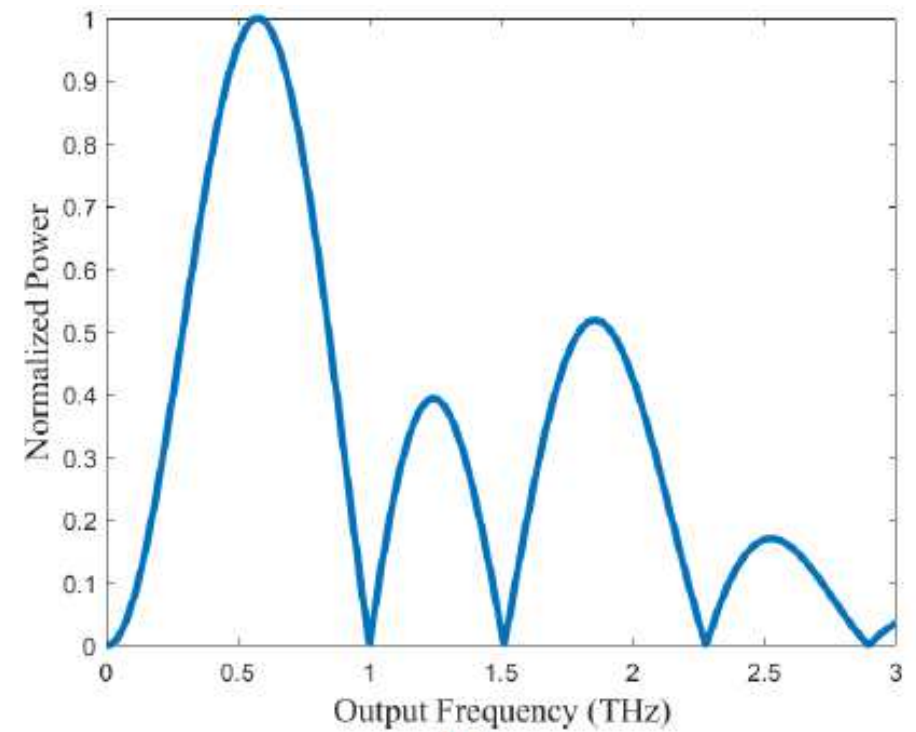

(a)

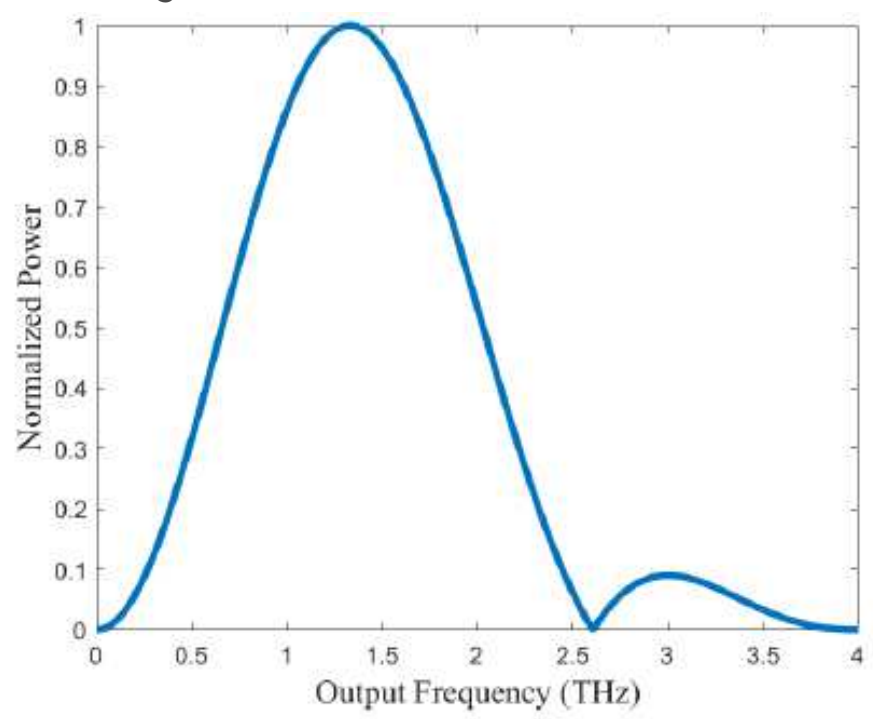

(b) 


\section{Figure 7}

Simulated frequency spectra for the transient arc model. (a) Frequency spectrum of the $0.5 \mathrm{THz}$ system. A peak occurs at $0.57 \mathrm{THz}$ with secondary peak occurring at $1.8 \mathrm{THz}$. A tertiary peak occurs at $1.25 \mathrm{THz}$, previously not predicted by the dipole model. (b) Frequency spectrum of the $1 \mathrm{THZ}$ system. A peak occurs at $1.33 \mathrm{THz}$ with a secondary peak at $3 \mathrm{THz}$.

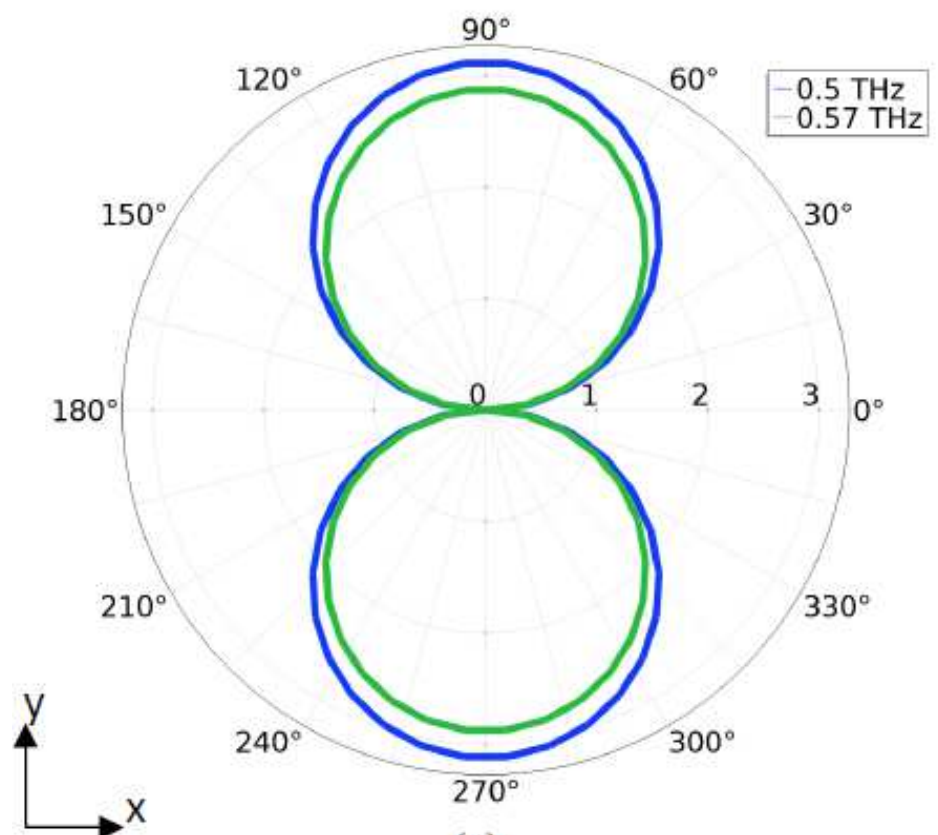

(a)

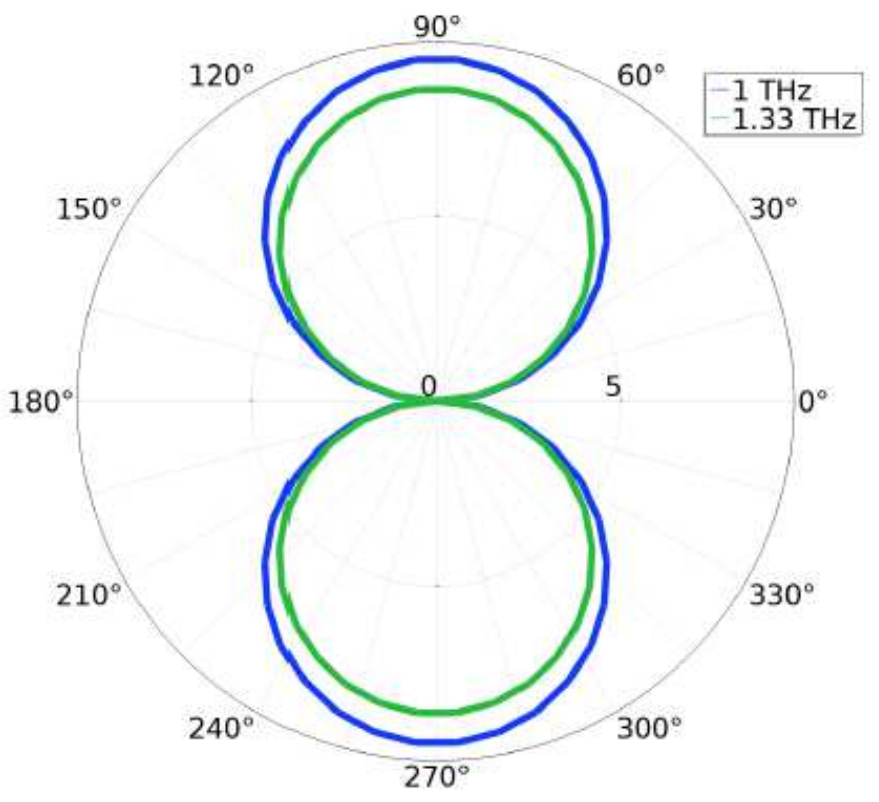

(b)

\section{Figure 8}

Simulated radiation patterns for the transient arc models in the orbital plane (arbitrary scale). (a) The 0.5 $\mathrm{THz}$ system and the $1 \mathrm{THz}$ system (b) at the design frequency (blue) and at peak emission (green). The finite element software used does not account for particle dynamics and hence does not produce the expected circular rotating dipole radiation pattern. The shape instead resembles a dipole oscillating along the $x$-axis. 


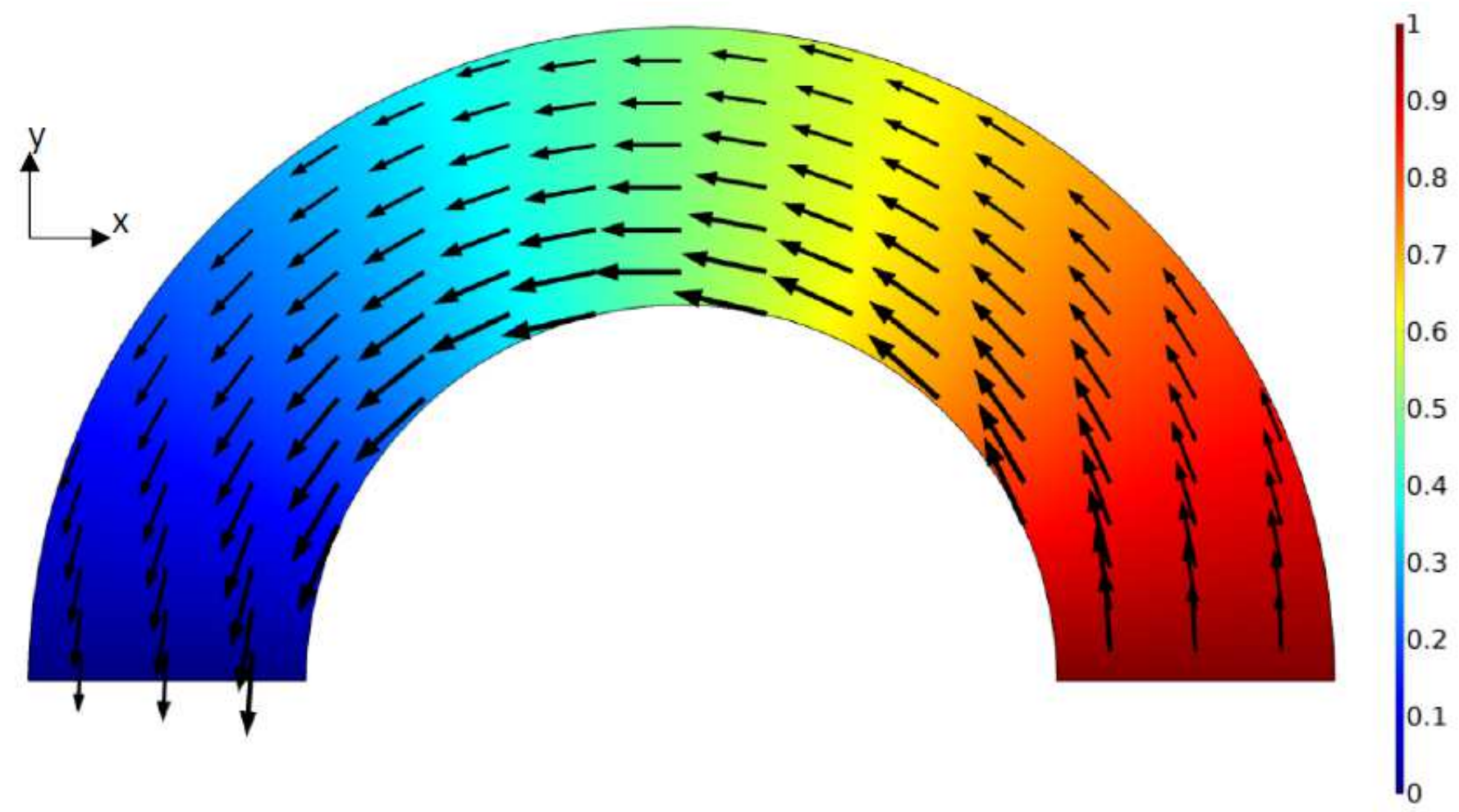

Figure 9

Simulated current density under DC bias of $1 \mathrm{~V}$ potential difference between source and drain. In both systems, current flows along the arc with the highest current density at the inner radius as indicated by arrow size. This is unlike the point charge assumption in which all of the charge located at the inner radius. 


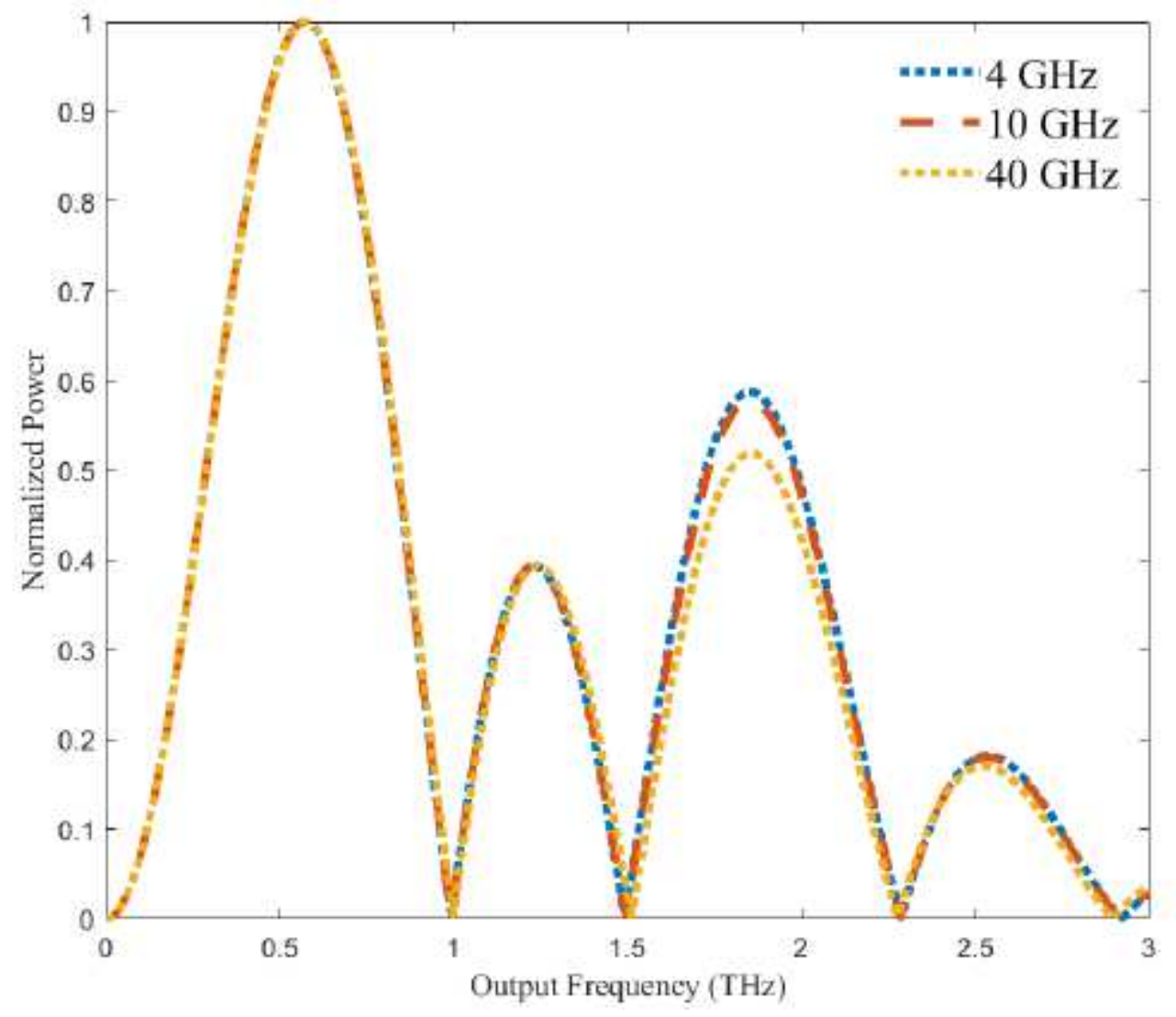

Figure 10

Effect of stimulus frequency on emission spectrum with $4 \mathrm{GHz}, 10 \mathrm{GHz}$, and $40 \mathrm{GHz}$ stimuli. Normalized spectra over the set of applied stimuli for the $0.5 \mathrm{THz}$ design. For all stimuli, the shape is consistent. The $4 \mathrm{GHz}$ and $10 \mathrm{GHz}$ slightly overshoot the $40 \mathrm{GHz}$ secondary peak at $1.8 \mathrm{THz}$. 